\title{
Communication Analysis through Visual Analytics: Current Practices, Challenges, and New Frontiers
}

This paper was downloaded from TechRxiv (https://www.techrxiv.org).

\section{LICENSE}

CC BY-NC-SA 4.0

SUBMISSION DATE / POSTED DATE

20-07-2021 / 26-07-2021

\section{CITATION}

Fischer, Maximilian T.; Dennig, Frederik L.; Seebacher, Daniel; Keim, Daniel A.; El-Assady, Mennatallah (2021): Communication Analysis through Visual Analytics: Current Practices, Challenges, and New Frontiers. TechRxiv. Preprint. https://doi.org/10.36227/techrxiv.15022791.v1

DOI 


\title{
Communication Analysis through Visual Analytics: Current Practices, Challenges, and New Frontiers
}

\author{
Maximilian T. Fischer, Frederik L. Dennig, Daniel Seebacher, Daniel A. Keim, and Mennatallah El-Assady
}

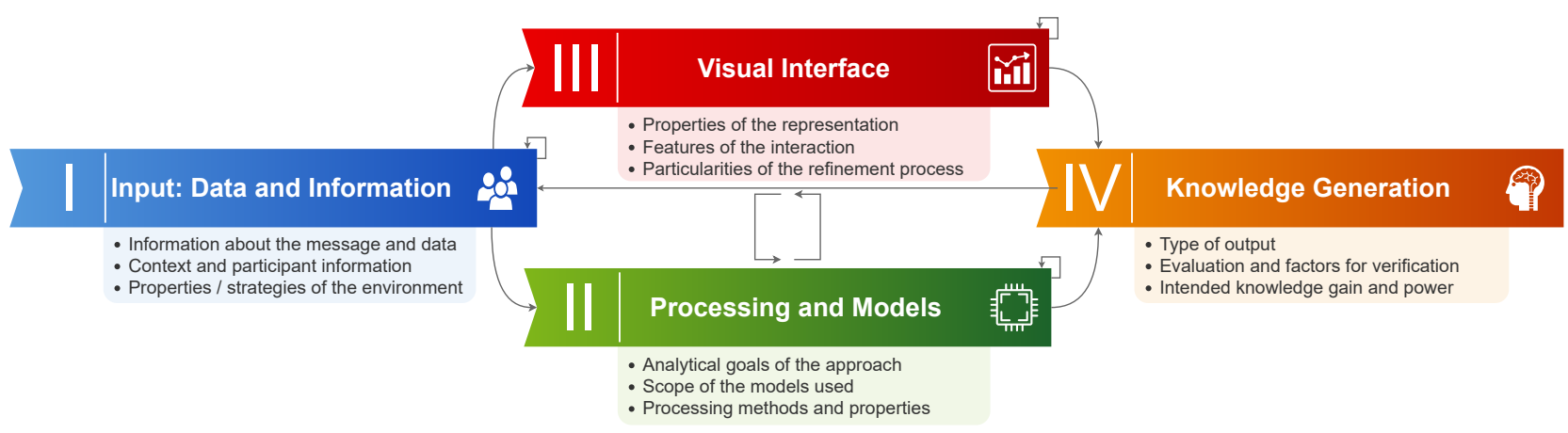

Fig. 1. Four main dimensions of our conceptual framework for communication analysis systems along with their characterization, which is based on the visual analytics process model by Keim et al. [1] and adapted by us to the communication analysis domain.

\begin{abstract}
The automated analysis of digital human communication data often focuses on specific aspects like content or network structure in isolation, while classical communication research stresses the importance of a holistic analysis approach. This work aims to formalize digital communication analysis and investigate how classical results can be leveraged as part of visually interactive systems, which offers new analysis opportunities to allow for less biased, skewed, or incomplete results. For this, we construct a conceptual framework and design space based on the existing research landscape, technical considerations, and communication research that describes the properties, capabilities, and composition of such systems through 30 criteria in four analysis dimensions. We make the case how visual analytics principles are uniquely suited for a more holistic approach by tackling the automation complexity and leverage domain knowledge, paving the way to generate design guidelines for building such approaches. Our framework provides a common language and description of communication analysis systems to support existing research, highlights relevant design areas while promoting and supporting the mutual exchange between researchers. Additionally, our framework identifies existing gaps and highlights opportunities in research areas that are worth investigating further. With this contribution, we pave the path for the formalization of digital communication analysis through visual analytics.
\end{abstract}

Index Terms-Communication analysis, visual analytics, conceptual framework, design space, state-of-the-art.

\section{INTRODUCTION}

$\mathrm{H}$ UMAN communication has been fundamentally transformed within the digital era. While digital forms of communication have slowly increased in the second half of the last century, the pace has fundamentally accelerated within the last two decades. Nowadays, digital communication is the norm rather than the exception, offering cost-effectiveness and instant access anywhere, leading to a change in communication behavior [2], [3].

With this transformation to digital communication [4], new research opportunities have emerged in a wide variety of different domains, ranging from engineering to social sciences to business. For example, it has been studied how visualization can show the evolution of dynamic communication networks [5], how discourse

- M. T. Fischer, F. L. Dennig, D. Seebacher, D. A. Keim, and M. El-Assady are with the University of Konstanz. E-mail: \{max. fischer, frederik.dennig, daniel.seebacher, keim, mennatallah.el-assady\}@uni-konstanz.de.

Manuscript received $x x x x x, x x x x$; revised $x x x x x, x x x x$. analysis for digital communication can be enhanced [6], or how team performance in business settings can be evaluated based on communication behavior [7]. For such analyses, digital analysis methods are often used to aid and support the (semi-)manual, domain-specific research methodologies.

In this paper, we focus on the field of interactive human communication analysis and specifically on automated and interactive communication analysis systems that primarily target written human communication (in the following: communication analysis systems). For the purpose of this paper, we define these systems as largely automated applications that also employ visual components to interactively analyze digital human communication. Most commonly, this can be chats, e-mail, etc. We do not consider approaches that focus primarily on the content itself (e.g., only sentiment analysis on a document collection alone), but the approach must thereby focus on distributed information exchanges by several participants. Consequently, public exchanges 
(e.g., Twitter or forums), even though they are somewhat related, do not fit directly as all information is available to all participants directly after a post (similar to a single group communication).

For such communication analysis systems, we notice a peculiar oddity: As we highlighted in previous work [8], and summarize in this paragraph shortly, the research into such systems is split into two fractions: the vast majority of existing approaches focus on either the content of communication or on the network aspect in isolation instead of taking into account the fundamental dynamics holistically. In contrast, this distinction between content and network is not present in the seminal works on human communication research [9], [10], [11]. Neither does it occur in more recent textbooks [12] or in current communication research [7], [13]. Even when digital methods are used to aid the manual analysis, for example, in psychology, often a holistic view is taken. Indeed, the holistic analysis of the network structure, the communication patterns as well as the content plays an integral part [11], [14] in this research field. As such, the analysis of the content or the network/metadata aspect alone can sometimes lead to an incomplete or even biased view, skewing the analysis results. Using independent approaches often introduces discontinuities, makes tasks more complicated, requires more manual work, and makes the detection of cross-matches more difficult. However, the development of such approaches is difficult as human communication data can be very complex, consisting of meta-data, network relations, and content, including explicit and implicit connotations, with some meaning only evident when considering everything together and in context. Nevertheless, this comparisons highlights a gap in such communication analysis systems.

We have begun to address these shortcoming in previous work [8] by presenting a prototype for one of the first holistic approaches to communication analysis.

However, the research in such systems has not yet received enough interest yet, in part due to the fact that the area is not yet accurately and systematically described. The need for such a formalization has been recognized [15] in communication sciences, but so far the opportunities, challenges, and pitfalls have primarily been described from a application-oriented perspective in the social sciences. While an effort has recently been made to map digital communication systems as a whole [16], the focuses was largely on content, infrastructure, and policy aspects, while leaving out the analysis and technical aspects. The same is true for the classical communication analysis research [9], [10], [11], [12]. One can base considerations on these historic works, but on the one hand it involves many aspects with no direct counterpart in digital communication. For example, facial expressions and gestures are not retained and accessible for the recipient of written exchanges. On the other hand and on a more fundamental level, digital communication has also transformed the way we communicate and the modalities we use [17] Digital communication has its own particularities and characteristics, which are, naturally, not discussed in the seminal works. However, when we instead focus on approaches describing actual systems including the technical aspects, many findings from communication research like the importance of a holistic view are not heeded. Instead, the vast majority of approaches fall into the two fractions discussed above, focusing either on concepts from natural language processing [18] for content analysis or discussing the field of social network analysis [19] for the network aspects.

In this work, we want to bridge the gap between communication research and modern communication analysis system development.
As evident in a few academic works [8], [20], [21], [22] and recent commercial systems [23], [24], [25], visualization and interactive user steering is a promising way [26] to begin to tackle the gap between the two different analysis modalities. So far, the field of communication analysis systems is not clearly defined from a scientific, technical perspective, lacking a common language to describe it. Consequently, so far, it has not been systematically explored how visual analytics principles are - and could be employed in communication analysis, how such systems can be described and categorized, for example, by their data, methodology, and information handling, and what a relevant taxonomy would look like. This lack of formalization makes developing such systems complicated and hinders comparison between approaches.

As part of this work, we aim to formalize digital communication analysis by constructing a design space of interactive digital communication analysis systems, making the following contributions:

- The creation of a conceptual framework of communication analysis systems, based on communication research, technical considerations, and systematically reviewing the properties of existing approaches and the associated tasks.

- A survey and comparison of existing approaches to assess their maturity and coverage, using our framework.

- A discussion on the open challenges and a list of future research opportunities on communication analysis systems.

Our work fills the gap in the formalization of communication analysis systems. With this contribution, we aim to better guide the research when assessing and developing approaches for communication analysis using visual analytics principles.

\section{BACKGROUND}

Communication analysis can use a variety of different techniques to analyze communication behavior. Like communication itself is an interactive exchange between sender and receiver, its analysis is not bound by a strict set of instructions. It builds upon the iterative analysis and progressive understanding of the meaning of the communication and its context, which makes automation difficult. As such, the area of semi-automated communication analysis fits very well with the concept of visual analytics, which is also based on the concept of iterative and interactive knowledge generation. In the following, we first describe the previous work in human sensemaking using visual analytics principles and how it integrates with communication analysis as well as the origins and main concepts of communication analysis itself.

\subsection{Visual Analytics and Knowledge Generation}

Visual analytics [1] describes the concept of combining human analytical reasoning with computational data analysis through interactive visualizations to gain knowledge. This knowledge generation process has been formalized by Sacha et al. [27] by explicitly describing the human cognition as three circular loops that build on each other: (1) exploration, (2) verification, and (3) knowledge generation. In the first loop (exploration), the user explores the data and the initial results the system presents. In communication analysis, this could, for example, encompass tasks such as finding the communication participants most central in a network or find those messages which contain specific semantic concepts. In the second loop (verification), a confirmatory analysis 
is conducted to either confirm or reject a hypothesis. Specific tasks here could include comparison between different results, further inspection, or aggregation. At last, in the third loop (knowledge generation), the user gains new knowledge and insights about the data and the analysis. Every step in this iterative process is essential, as it allows the analysts to form connections between different analysis modalities. This is especially important, because these different modalities are very common in communication analysis [28], which makes visual analytics especially suited for a holistic, non-specialized analysis.

\subsection{Communication Analysis}

The origins of communication research can be traced backed to ancient times, with the study of rhetoric and oratory as well as persuasion in ancient Greece and Rome. However, the formal study of communication as a process did not really start until the early 20th century. Of particular importance in this early phase are the works of Cooley [29] and Lippmann [30], which provided one of the first formal definitions of communication and established the influence of social aspects in communication, as well as the work of Simmel [9], studying social relationships and their influence. These human interrelations were further studied by Moreno [10], credited with laying the foundations for social network analysis (SNA). These early works were then further extended by Bavelas [31] and Leavitt [32], who introduced a taxonomy for communication patterns in groups. Only a few years later, Savage and Deutsch [14] paved the way to analyze communication through computer aided modeling.However, manual analysis and research was still the leading methodology. In the coming years, Watzlawick et al. [11] developed a theory of communication, while other work [33] further described particularities.Around this time, the field was further extended by Roger [34] and adapted to the modern understanding of communication networks. Recent works highlight the main findings [12].

With the advent of digital processing and computational power came the shift from laboursome manual analysis [35] first to digitally supported [36] and later to highly automated analysis. All three levels are fully justified and cover important areas in their fields. However, it is noticeable, while not completely surprising, that the completeness of the analysis developed in the opposite direction of automation level, with solutions being ever more specialized and focusing on just specific aspects the more they are automated. For example, modern systems allow us to analyze communication behavior and social ties using centrality measures [37] or describe complete artificial networks in social sciences [38]. Specialized toolkits have been developed to analysis such network structures, like Pajek [39] or Gephi [40]. All these approaches primarily focus on the network aspects, omitting most of the meta-data and especially the content. Other approaches instead focus only on it, like keyword-based searches [41] to filter communication for certain content, or aiming to improve the understanding of the meaning of communication using concepts such as sentiment analysis [42] or topic modeling [43].

From a visual perspective, several approaches primarily follow a node-link-diagram-based approach, like Gephi [40], and many commercial solutions like IBM's i2 Analyst's Notebook [44], Pajek [39], Palantir Gotham [24], DataWalk [25], and Nuix Discover and Nuix Investigate [23]. Another class of approaches uses matrixbased approaches to analyze the communication relations, for example, MatrixExplorer [45] or NodeTrix [46]. Another set of approaches uses timeline designs like CloudLines [47], while others like Fu et al. [48] modify graph presentations through multiple planes. For a detailed discussion, we refer to previous work [8], where we discuss the existing visual analytics research landscape for communication analysis systems extensively.

\section{Methodology}

The goals and methods employed in communication analysis are diverse and manifold, as illustrated by the broadness of existing approaches. In the following, we aim towards tackling the central question of a common description of communication analysis: How can the different approaches in communication analysis systems be described within a common, conceptual framework?

Framework Basis - We propose to base such a framework on three areas of consideration: (1) Communication research offers decades of research on the particularities of (often nontechnical) communication analysis. For this work, we integrate concepts primarily from later summary works [11], [12], [33]. We discuss the concepts and ideas which are based on communication research in detail in the relevant parts of Section 4. (2) Technical considerations of possible approaches allow for production-based selection criteria, taking into account design properties such as analyzable data types, data representation, and flow, or limitations like scalability from a technical standpoint. These are closely linked to the last category. (3) The existing research landscape of interactive communication analysis systems provides a foundation for the classification of approaches based on measures such as analytical goals, visualization and interaction methods, or the power of the knowledge generation process. While the first two considerations are references directly when describing the framework in Section 4 the third requires a thorough preparation:

Seed Papers for Existing Research Landscape - To analyze state-of-the-art communication analysis systems and contribute a first set of classification criteria, we conducted a seed literature survey to get an initial idea of a large set of existing approaches.

First, we collected papers using a keyword-based search, and as we are considering only approaches from visual data analysis, restrict to the following high-quality journals and conferences:

- IEEE Transactions of Visualization and Computer Graphics (TVCG, including IEEE VIS proceedings)

- Computer Graphics Forum (EuroVis and EuroVA)

We consider approaches published in the last 15 years (from 2006 to 2021) to focus on recent developments for all venues. Note that we intentionally restricted our search space to these venues, considering only well known information visualization publications. The high number of initial approaches and the high discard rate for $\mathrm{CHI}$ is due to the abundant use of the phrase communication when referring to user actions. Also, while several approaches discuss relevant communication analysis aspects (like analogical code, e.g. [49]), many approaches published at $\mathrm{CHI}$ discuss semi-manual analysis or user studies (e.g., [50], [51], [52], [52], [53]) and only few describe analysis systems. This step from a theoretical description of the properties in one area to the actual conceptualization of an semi-automated system that can perform such tasks is often still missing. It is the aim of this design space, to also identify such areas (see also Section 5.1. As such, the goal of this paper-not being a survey-is not to construct a complete survey of approaches - we aim for a large and diverse enough sample to make justifiable conclusions (for 


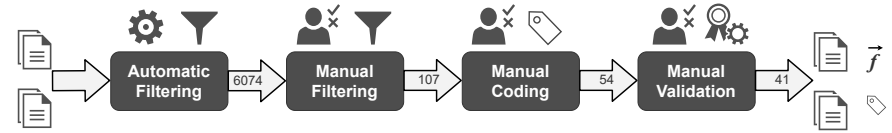

Fig. 2. The paper collection and coding process. It consists of four main steps: (1) Automated filtering, (2) manual filtering, (3) manual coding, and (4) manual validation.

\begin{tabular}{lcccc}
\hline Venue & \#Coll. & \#Filtered & \#Coded & \#Final \\
\hline IEEE TVCG & 790 & 35 & 27 & 23 \\
Computer Graphics Forum & 495 & 17 & 11 & 8 \\
CHI Proceedings & 4789 & 49 & 10 & 4 \\
Commercial Systems & - & 6 & 6 & 6 \\
\hline Total & 6074 & 107 & 54 & $\mathbf{4 1}$ \\
\hline
\end{tabular}

TABLE 1. Publications per venue and paper count for collection step.

a more detailed discussion and a possible future survey, see also Section 57. Indeed, we are well aware that other approaches exists, being published in other venues (e.g., [48], [54]). Also, the survey on text visualization by Kucher et al. [55], especially the group discourse analysis, provides some further approaches that have been published in IUI [56], [57] or TiiS [58]. Further, other venues like PacificVis [48], [59] or domain specific journals like Digital Investigation [20] also contributed valid approaches. The inclusion of all these approaches would go beyond the scope of this paper, given its size constraints.

To broaden the perspective, in a second step, we consulted with eight domain experts about approaches used in practice The experts belong to the field of law enforcement, working for various European law enforcement agencies, and each has extensive experience with digital investigations, including communication analysis, working in the field from ten to over 30 years. They contributed a collection of six actual systems [23], [24], [25], [39], [40], [44] applied in the field (including commercial). Those approaches were included in the Selection Methodology from Step 2 onward and were not simultaneously discovered during the seed paper selection. However, some approaches [40], [44] can be considered to be universally known in the community.

Selection Methodology - For the actual paper selection methodology, we follow a four-step approach (see also Figure 2 and Table 11. First, we conducted a keyword-based seed search for the words communication and analysis on the titles, abstract, index terms, and contents of publications in each of the venues described above. Secondly, we went through all these papers' titles and abstracts manually, discarding those which clearly are not concerned with communication analysis systems, reducing the selection significantly. In this step, we included approaches suggested by the domain experts. Third, we manually looked at the remaining papers and decided if they indeed describe a communication analysis system. In the final step, we validated the results by checking borderline cases, consequently removing seven papers. Or final collection includes 41 approaches.

\section{Conceptual Framework}

In the following section, we aim to construct a possible conceptual framework that encompasses discerning aspects of communication analysis systems. As with any taxonomy, the development took dozens of iterations, and we cannot cover its complete evolution here. Instead, we highlight the main considerations and, for each property, give a justification. For the complete conceptual framework, see Figure 33. In the following, we focus on the argumentation and reasoning, categorizing the existing approaches just in an exemplary manner. For the full classification of the selected approaches from above, consult Table 2

Main Considerations - When designing a conceptual framework, the structuring methodology is of central importance. One standard methodology is to use a task-based grouping. However, when analyzing the different approaches, we realized that sometimes very different methods are employed for the same task. For example, to discover key persons within a communication network, one can either use an SNA-based approach using nodelink visualizations and centrality measures or instead leverage a geometric deep learning model that is interactively visualized using a matrix-based method. These methods have very different side effects, visualization, and interaction techniques and make very different assumptions and requirements on the data.

The primary goal is to design a conceptual framework of communication analysis in visual analytics from a communication theoretical standpoint, that is easily adapted and applicable. Therefore, we base our framework on the established visual analytics process model by Keim et al. [1] to cover the main areas. We slightly modify the terminology, proposing four main dimensions, which are characterized further in Figure 11. (I) Input: Data and Information (4.1), (II) Processing and Models (4.2), (III) Visual Interface (4.3), and (IV) Knowledge Generation 4.4).

In our work, we develop these dimensions specifically for communication analysis. To achieve this, we include properties relevant in communication research as well as discerning technical properties, based on the existing research landscape. In the following, we discuss each dimension individually. As a note of caution, we stress that many properties we present in the following are themselves multifaceted and, for a detailed analysis, benefit from a more nuanced discussion in more detail than we can include within the limited space available here.

\subsection{Input: Data and Information}

This category focuses on information, context and environment of the communication. In contrast to the process model by Keim et al. [1], we specifically understand this category to focus on the information theoretical aspects and the collection process itself, not so much on any preprocessing steps. As such, the structuring is partly based on classical communication research [11], [12], [33], discussing the content and meaning, context, and relationship aspects of communication extensively. Building on this, we propose three main subcategories: message, communication participant, and environment. We complement these with properties relevant for digital systems, like the data type and collection properties.

\subsubsection{Message}

Communication can be regarded as the exchange of symbols. In computer science, message is more common. The main considerations are the entailed meaning and how it is transported:

Data Type - The data type refers to the content type of the data from a technical point of view. When looking at data classification in information visualization [88], we can identify several data types which are relevant for communication analysis: text, audio, image/video, network, and time-series. Based on the usage in current approaches (see Section 3), the two most relevant ones are text data (e.g., [69]) and network (structure) data 


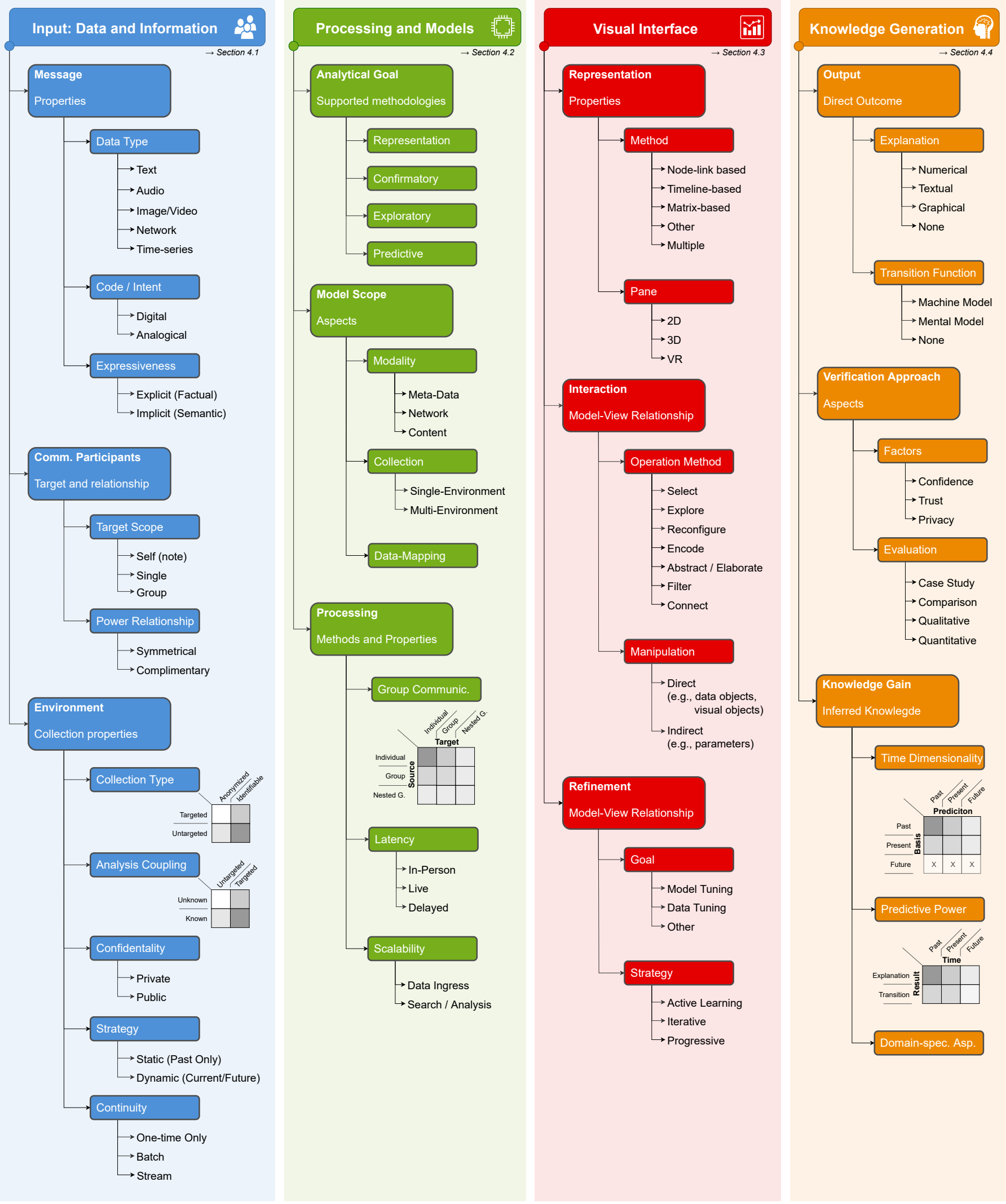

Fig. 3. Our conceptual framework of communication analysis systems. It consists of the four main dimensions Input: Data and Information, Processing and Models, Visual Interface, and Knowledge Generation, described in more detail in Section 3 and also in Figure 1 Each category contains several properties and sub-properties, which allow for systematical analysis of such systems. Note that the graphic highlights some of the most relevant aspects for individual properties, which can be used for a simplified comparison. However, the properties themselves are multifaceted and, for a detailed analysis, should be discussed more nuanced and in more detail than indicated by these examples. 
TABLE 2. Communication analysis system classification summarizing the different properties of the approaches. The classification criteria are formed on a subset based on the conceptual framework we developed in Section 4 and which is shown in Figure 3 The selected approaches and most categories are also available on a dedicated website at communication-analysis.dbvis.de (for details, see Section 5.2 .

Generic Properties: Approach supports a specific property or not $\bigcirc$, or support is only very basic or limited (e.g., show as associated entry without analysis). $\dagger$ : Indicates approaches which are commercial systems or widely used in the industry.

The following encodings have special symbols:

Strategy: The approach supports a static $\mathbf{S}$ or a dynamic $\mathbf{D}$ environment

Continuity: The approach supports either a one-time only data loading $\mathbf{O}$, iterative batches of data $\mathbf{B}$ or a data stream $\mathbf{S}$.

Collection: The approach supports either the analysis of single environments $\mathbf{S}$ or of multiple environment $\mathbf{M}$ simultaneously.

Group Communication: The approach supports different types of group communication analysis, as encoded in a $3 \times 3$ matrix. The rows (source) and columns (target) specify individual, group, and nested groups, respectively. For example, encodes that the approach supports only the analysis of communication between individuals and, additionally, of individual to groups. Groups as source are not supported and nested groups not at all.

Latency: The approach supports either the analysis of in-person communication $\mathbf{I}$, the live $\mathbf{L}$ analysis of communication or a delayed $\mathbf{D}$ analysis a posteriori.

Scalability: The approach supports analysis of tens (I), thousands (II), or millions (IIII) of cases for analysis.

Evaluation: Case study or example $\boldsymbol{E}$, comparison with other techniques $\leftrightarrow$, and expert study $\bullet$

Time Dimensionality: The approach supports different time dimensionalities, as encoded in a $2 \times 3$ matrix. The basis of the knowledge is encoded in the rows (either past or present),

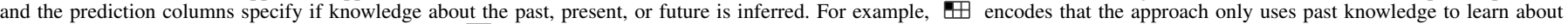
the past, e.g., to find a historic pattern. The encoding $\$$ instead indicates the approach uses past and present data to predict future developments.

Predictive Power: The approach's predictive power encodes in a $2 \times 3$ matrix the type of output (explanation or transition, as rows) in relation to the time dimensionalities past, present, and future, as columns. For example, $\mathbb{Z}$ encodes a explanation about the past, while $\mathbf{m}$ can also give explanations about the future. Instead, the encoding indicates that the approach supports both a explanation but also a transition function to explain past as well as classify present and predict future developments.

\begin{tabular}{|c|c|c|c|c|c|c|c|c|c|c|c|c|c|c|c|c|c|c|c|c|c|c|c|c|c|c|c|}
\hline & & & 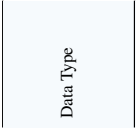 & 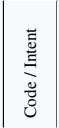 & 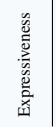 & 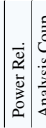 & 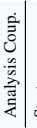 & 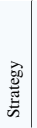 & 鳏 & 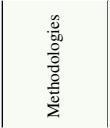 & 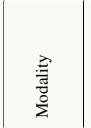 & בे & & $\begin{array}{l}0 \\
\text { 产 } \\
\text { o }\end{array}$ & 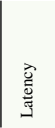 & 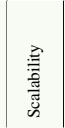 & 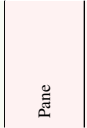 & 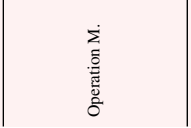 & $\begin{array}{l}\text { 总 } \\
\text { 言 } \\
\text { 言 } \\
\text { 言 }\end{array}$ & $\begin{array}{l}\overline{8} \\
\text { S̆ }\end{array}$ & 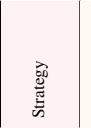 & 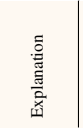 & 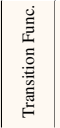 & 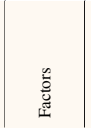 & 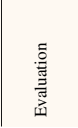 & 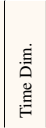 & 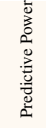 \\
\hline & Approach & 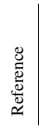 & 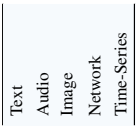 & 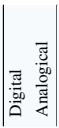 & 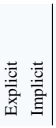 & 悹 & & 它 & $\mid \frac{\tilde{\alpha}}{\partial}$ & 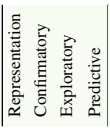 & 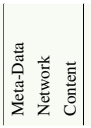 & 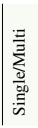 & $\mid$ & 总 & $\stackrel{\text { Ṡ }}{S}$ & 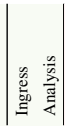 & तิ ले & 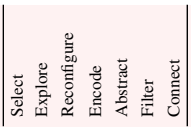 & $\mid$ & 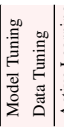 & 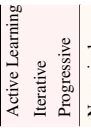 & 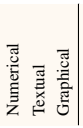 & 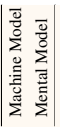 & 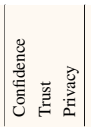 & $\sum_{\tilde{k}}^{\tilde{k}}$ & 崖 & 惫 \\
\hline & \begin{tabular}{|l} 
Pajek $^{\dagger}$ \\
Gephi $^{\dagger}$ \\
SaNDVis \\
Ghani et al. \\
Elzen et al. \\
NEREx \\
i2 Analyst's NB \\
Palantir Gotham \\
DataWalk $^{\dagger}$ \\
Nuix D./L.
\end{tabular} & \begin{tabular}{|l|}
39 \\
40 \\
60 \\
61 \\
62 \\
62 \\
63 \\
44 \\
24 \\
25 \\
23 \\
\end{tabular} & $\left|\begin{array}{lllll}0 & 0 & 0 & \bullet & 0 \\
0 & 0 & 0 & \bullet & 0 \\
0 & 0 & 0 & \bullet & 0 \\
0 & 0 & 0 & \bullet & 0 \\
0 & 0 & 0 & \bullet & 0 \\
0 & 0 & 0 & 0 & 0 \\
0 & 0 & 0 & \bullet & 0 \\
0 & 0 & 0 & 0 & 0 \\
0 & 0 & 0 & 0 & 0 \\
0 & 0 & 0 & 0 & 0\end{array}\right|$ & $\left|\begin{array}{ll}0 & 0 \\
0 & 0 \\
0 & 0 \\
0 & 0 \\
0 & 0 \\
0 & 0 \\
0 & 0 \\
0 & 0 \\
0 & 0\end{array}\right|$ & $\begin{array}{ll}\bullet & 0 \\
0 & 0 \\
0 & 0 \\
0 & 0 \\
0 & 0 \\
0 & 0 \\
0 & 0 \\
0 & 0 \\
0 & 0\end{array} \mid$ & $\mid$\begin{tabular}{l|l}
0 & $c$ \\
0 & $c$ \\
0 & $c$ \\
0 & $c$ \\
0 & $c$ \\
0 & $c$ \\
0 & $c$ \\
0 & $c$ \\
0 & 0 \\
0 & $c$
\end{tabular} & $\begin{array}{lll}0 & 1 \\
0 & 1 \\
0 & 1 \\
0 & 1\end{array}$ & $\begin{array}{l}\text { S } \\
\text { S } \\
\text { S }\end{array}$ & \begin{tabular}{|l|} 
\\
$B$ \\
$B$ \\
\\
$B$ \\
$B$ \\
$S$ \\
$B$
\end{tabular} & & $\left|\begin{array}{lll}0 & 0 & 0 \\
0 & \bullet & 0 \\
0 & 0 & 0 \\
0 & 0 & 0 \\
0 & 0 & 0 \\
0 & 0 & 0 \\
0 & \bullet & 0 \\
0 & \bullet & 0 \\
0 & \bullet & 0 \\
0 & 0 & 0\end{array}\right|$ & \begin{tabular}{|l}
$\mathbf{S}$ \\
$\mathbf{S}$ \\
$\mathbf{M}$ \\
$\mathrm{M}$ \\
$\mathrm{M}$ \\
$\mathrm{M}$
\end{tabular} & $\begin{array}{l}0 \\
0 \\
0 \\
0 \\
0\end{array}$ & $\begin{array}{l}\text { 田 } \\
\text { 田 } \\
\text { 田 } \\
\text { 田 } \\
\text { 田 } \\
\text { 田 } \\
\text { 田 } \\
\text { 田 }\end{array}$ & \begin{tabular}{|c}
$\mathbf{D}$ \\
$\mathbf{D}$ \\
$\mathbf{D}$ \\
$\mathbf{D}$ \\
$\mathbf{D}$ \\
$\mathbf{D}$ \\
$\mathbf{D}$ \\
$\mathbf{L} \mathbf{D}$ \\
$\mathbf{D}$ \\
$\mathrm{L}$ \\
\end{tabular} & $\left|\begin{array}{ccc}\text { IIII } & \text { II } \\
\text { IIII } & \text { II } \\
\text { IIII II } \\
\text { II } & \text { II } \\
\text { II } & \text { II } \\
\text { IIII } & \text { II } \\
\text { IIII } & \text { I } \\
\text { IIII } & \text { I } \\
\text { IIII } & \text { I } \\
\text { IIII } & \text { II }\end{array}\right|$ & $\left|\begin{array}{lll}\bullet & \bullet & 0 \\
0 & 0 & 0 \\
0 & 0 & 0 \\
\bullet & 0 & 0 \\
\bullet & 0 & 0 \\
0 & 0 & 0 \\
0 & 0 & 0 \\
\bullet & 0 & 0 \\
\bullet & 0 & 0 \\
0 & 0 & 0\end{array}\right|$ & $\mid \begin{array}{lllll}\bullet & \bullet & \bullet & 0 & \bullet \\
\bullet & \bullet & \bullet & 0 & \bullet \\
\bullet & \bullet & \bullet & 0 & \bullet \\
\bullet & \bullet & \bullet & 0 & \bullet \\
\bullet & \bullet & \bullet & 0 & \bullet \\
\bullet & \bullet & \bullet & \bullet & \bullet \\
\bullet & \bullet & \bullet & \bullet & \bullet \\
\bullet & \bullet & \bullet & \bullet & \bullet \\
\bullet & \bullet & \bullet & \bullet & \bullet \\
\bullet & \bullet & \bullet & \bullet & \bullet\end{array}$ & $\left|\begin{array}{ll}\bullet & 0 \\
\bullet & \bullet \\
\bullet & \bullet \\
\bullet & \bullet \\
\bullet & \bullet \\
\bullet & \bullet \\
\bullet & \bullet \\
\bullet & \bullet \\
\bullet & \bullet \\
\bullet & \bullet\end{array}\right|$ & $\left|\begin{array}{lll}0 & \bullet \\
0 & \bullet \\
0 & \bullet \\
0 & \bullet \\
0 & \bullet \\
0 & \bullet \\
0 & \bullet \\
0 & \bullet \\
0 & \bullet \\
\bullet & \bullet & 0\end{array}\right|$ & $\left|\begin{array}{lll}0 & \bullet & \bullet \\
0 & \bullet & \bullet \\
0 & \bullet & \bullet \\
0 & \bullet & \bullet \\
0 & \bullet & \bullet \\
\bullet & \bullet & \bullet \\
\bullet & \bullet & \bullet \\
\bullet & \bullet & \bullet \\
\bullet & \bullet & 0 \\
0 & \bullet & 0\end{array}\right|$ & $\left|\begin{array}{lll}\bullet & 0 & \bullet \\
\bullet & 0 & \bullet \\
0 & 0 & \bullet \\
0 & 0 & \bullet \\
\bullet & 0 & \bullet \\
0 & \bullet & \bullet \\
\bullet & \bullet & \bullet \\
\bullet & \bullet & \bullet \\
\bullet & \bullet & \bullet \\
\bullet & \bullet & \bullet\end{array}\right|$ & $\left|\begin{array}{ll}0 & \bullet \\
0 & \bullet \\
0 & \bullet \\
0 & \bullet \\
0 & \bullet \\
0 & \bullet \\
\bullet & \bullet \\
\bullet & \bullet \\
0 & \bullet \\
\bullet & \bullet\end{array}\right|$ & $\left|\begin{array}{lll}0 & 0 & 0 \\
0 & 0 & 0 \\
0 & 0 & 0 \\
0 & 0 & 0 \\
0 & 0 & 0 \\
0 & 0 & 0 \\
0 & 0 & 0 \\
0 & 0 & 0 \\
0 & 0 & 0 \\
0 & 0 & 0\end{array}\right|$ & $\begin{array}{c}\boldsymbol{\theta} \\
\boldsymbol{\theta} \\
\boldsymbol{\theta} \\
\dot{-} \\
\boldsymbol{\theta} \\
\boldsymbol{\theta} \\
- \\
- \\
- \\
- \\
-\end{array}$ & 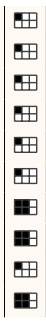 & 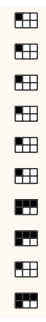 \\
\hline & \begin{tabular}{|l|} 
Reccurence Plots \\
GestaltMatrix \\
MatrixWave \\
SmallMultiPiles \\
HyperMatrix \\
\end{tabular} & \begin{tabular}{|l|}
64 \\
65 \\
59 \\
66 \\
67 \\
\end{tabular} & $\left|\begin{array}{lllll}- & 0 & 0 & 0 & 0 \\
0 & 0 & 0 & \bullet & 0 \\
0 & 0 & 0 & 0 & \bullet \\
0 & 0 & 0 & \bullet & 0 \\
0 & 0 & 0 & \bullet & 0\end{array}\right|$ & $\left|\begin{array}{ll}0 & 0 \\
0 & 0 \\
0 & 0 \\
0 & 0 \\
0 & 0\end{array}\right|$ & $\left|\begin{array}{ll}\bullet & 0 \\
\bullet & 0 \\
\bullet & 0 \\
\bullet & 0 \\
\bullet & 0\end{array}\right|$ & $\left|\begin{array}{l}0 \\
0 \\
0 \\
0 \\
0\end{array}\right|$ & \begin{tabular}{l|l}
0 & 1 \\
0 & \\
0 & \\
0 & \\
0 & \\
0
\end{tabular} & \begin{tabular}{|l|}
$\mathbf{S}$ \\
$\mathbf{S}$ \\
$\mathrm{S}$ \\
$\mathrm{S}$ \\
$\mathrm{S}$
\end{tabular} & $\left|\begin{array}{l}0 \\
0 \\
0 \\
0 \\
0\end{array}\right|$ & $\left|\begin{array}{llll}0 & \bullet & \bullet & 0 \\
0 & 0 & 0 & 0\end{array}\right|$ & $\left|\begin{array}{lll}0 & 0 & \bullet \\
0 & \bullet & 0 \\
0 & 0 & 0 \\
0 & \bullet & 0 \\
0 & \bullet & \bullet\end{array}\right|$ & \begin{tabular}{|l}
$\mathbf{S}$ \\
$\mathbf{S}$ \\
$\mathbf{S}$ \\
$\mathbf{S}$ \\
$\mathbf{S}$
\end{tabular} & $\begin{array}{l}0 \\
0\end{array}$ & $\begin{array}{l}\text { 田 } \\
\text { 田 } \\
\text { 田 }\end{array}$ & $\begin{array}{l}\mathrm{D} \\
\mathrm{D} \\
\mathrm{D} \\
\mathrm{D} \\
\mathrm{D}\end{array}$ & $\left|\begin{array}{ll}\text { II } & \text { II } \\
\text { II } & \text { I } \\
\text { II } & \text { I } \\
\text { II } & \text { II } \\
\text { II } & \text { I }\end{array}\right|$ & $\left|\begin{array}{lll}- & 0 & 0 \\
\bullet & 0 & 0 \\
\bullet & 0 & 0 \\
\bullet & 0 & 0 \\
\bullet & 0 & 0\end{array}\right|$ & $\left|\begin{array}{lllllll}\bullet & \bullet & \bullet & 0 & 0 & \bullet & \bullet \\
0 & \bullet & 0 & 0 & 0 & 0 & 0 \\
\bullet & \bullet & \bullet & 0 & 0 & \bullet & \bullet \\
\bullet & \bullet & \bullet & 0 & \bullet & \bullet & \bullet \\
\bullet & \bullet & \bullet & 0 & \bullet & \bullet & \bullet\end{array}\right|$ & $\left|\begin{array}{ll}0 & \bullet \\
0 & 0 \\
\bullet & \bullet \\
\bullet & \bullet \\
\bullet & \bullet\end{array}\right|$ & $\left|\begin{array}{ll}0 & 0 \\
0 & 0 \\
0 & 0 \\
0 & \bullet \\
0 & 0\end{array}\right|$ & $\left|\begin{array}{lll}0 & 0 & \bullet \\
0 & \bullet & 0 \\
0 & \bullet & \bullet \\
0 & \bullet & \bullet \\
0 & \bullet & \bullet\end{array}\right|$ & $\left|\begin{array}{lll}0 & \bullet & \bullet \\
0 & 0 & \bullet \\
0 & 0 & \bullet \\
0 & 0 & \bullet \\
\bullet & 0 & \bullet\end{array}\right|$ & $\left|\begin{array}{ll}0 & \bullet \\
0 & \bullet \\
0 & \bullet \\
0 & \bullet \\
\bullet & \bullet\end{array}\right|$ & $\left|\begin{array}{lll}0 & 0 & 0 \\
0 & 0 & 0 \\
0 & 0 & 0 \\
0 & 0 & 0 \\
0 & 0 & 0\end{array}\right|$ & 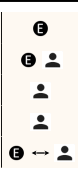 & 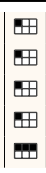 & $\begin{array}{l}\mathbb{H} \\
\Psi \\
\mathbb{H}\end{array}$ \\
\hline & \begin{tabular}{|l} 
Themail \\
TextFlow \\
CloudLines \\
DecisionFlow \\
OpinionFlow \\
Han et al. \\
Liu et al. \\
ThreadReconst. \\
T-Cal \\
netflower \\
WeSeer \\
\end{tabular} & \begin{tabular}{|l|}
68 \\
69 \\
47 \\
70 \\
21 \\
71 \\
72 \\
72 \\
73 \\
74 \\
75 \\
76 \\
\end{tabular} & $\left|\begin{array}{lllll}\bullet & 0 & 0 & 0 & \bullet \\
\bullet & 0 & 0 & 0 & \bullet \\
\bullet & 0 & 0 & 0 & \bullet \\
0 & 0 & 0 & 0 & \bullet \\
\bullet & 0 & 0 & 0 & 0 \\
0 & 0 & 0 & 0 & \bullet \\
\bullet & 0 & 0 & 0 & \bullet \\
\bullet & 0 & 0 & 0 & \bullet \\
\bullet & 0 & 0 & 0 & \bullet \\
\bullet & 0 & 0 & \bullet & \bullet \\
0 & 0 & 0 & 0 & \bullet\end{array}\right|$ & $\left|\begin{array}{ll}0 & 0 \\
\bullet & 0 \\
0 & 0 \\
0 & 0 \\
0 & 0 \\
0 & 0 \\
0 & 0 \\
0 & 0 \\
0 & 0 \\
0 & 0 \\
0 & 0\end{array}\right|$ & $\begin{array}{ll}\bullet & 0 \\
\bullet & 0 \\
\bullet & 0 \\
\bullet & 0 \\
\bullet & 0 \\
0 & 0 \\
\bullet & 0 \\
0 & \bullet \\
0 & 0 \\
0 & 0 \\
0 & 0\end{array} \mid$ & $\left|\begin{array}{l}0 \\
0 \\
0 \\
0 \\
0 \\
0 \\
0 \\
0 \\
0 \\
0 \\
0 \\
0 \\
0 \\
0\end{array}\right|$ & $\begin{array}{l}0 \\
0 \\
0 \\
0 \\
0 \\
0 \\
0 \\
0\end{array}$ & \begin{tabular}{l|}
$S$ \\
S \\
S \\
S \\
S \\
S \\
S \\
S \\
S \\
S \\
S \\
\end{tabular} & $\begin{array}{l}0 \\
0 \\
0 \\
0 \\
0 \\
0 \\
0 \\
0 \\
0 \\
0 \\
0 \\
\end{array}$ & $\begin{array}{l}0 \\
\bullet \\
\bullet \\
0\end{array}$ & $\left|\begin{array}{lll}0 & 0 & \bullet \\
\bullet & 0 & \bullet \\
\bullet & 0 & 0 \\
\bullet & 0 & 0 \\
0 & 0 & \bullet \\
0 & 0 & 0 \\
\bullet & 0 & \bullet \\
\bullet & 0 & \bullet \\
0 & 0 & \bullet \\
\bullet & 0 & 0 \\
0 & 0 & \bullet\end{array}\right|$ & \begin{tabular}{|l}
$\mathbf{S}$ \\
$\mathbf{S}$ \\
$\mathbf{S}$ \\
$\mathbf{S}$ \\
$\mathbf{S}$ \\
$\mathbf{S}$
\end{tabular} & $\begin{array}{l}0 \\
0\end{array}$ & $\mid \begin{array}{l}\text { 田 } \\
\text { 田 }\end{array}$ & $\begin{array}{l}\mathrm{D} \\
\mathrm{D} \\
\mathrm{D} \\
\mathrm{D} \\
\mathrm{D} \\
\mathrm{D} \\
\mathrm{D} \\
\mathrm{D} \\
\mathrm{D} \\
\mathrm{D} \\
\mathrm{D}\end{array}$ & $\left|\begin{array}{cc}\text { IIII } & \text { II } \\
\text { II } & \text { II } \\
\text { IIII } & \text { I } \\
\text { II } & \text { II } \\
\text { IIII } & \text { I } \\
\text { IIII } & \text { II } \\
\text { II } & \text { I } \\
\text { IIII } & \text { II } \\
\text { IIII } & \text { II } \\
\text { II } & \text { I } \\
\text { IIII } & \text { II }\end{array}\right|$ & $\begin{array}{lll}- & 0 & 0 \\
\bullet & 0 & 0 \\
0 & 0 & 0 \\
0 & 0 & 0 \\
0 & 0 & 0 \\
\bullet & 0 & 0 \\
0 & 0 & 0 \\
0 & 0 & 0 \\
0 & 0 & 0 \\
0 & 0 & 0 \\
0 & 0 & 0\end{array} \mid$ & $\begin{array}{llll}\bullet & \bullet & \bullet & 0 \\
\bullet & \bullet & \bullet & 0 \\
\bullet & \bullet & 0 & 0 \\
\bullet & \bullet & \bullet & 0 \\
\bullet & \bullet & 0 & 0 \\
\bullet & \bullet & \bullet & 0 \\
\bullet & \bullet & 0 & 0 \\
\bullet & \bullet & \bullet & 0 \\
\bullet & \bullet & \bullet & 0 \\
\bullet & \bullet & \bullet & 0 \\
\bullet & \bullet & \bullet & 0\end{array}$ & $\left|\begin{array}{ll}0 & 0 \\
0 & \bullet \\
0 & \bullet \\
\bullet & \bullet \\
\bullet & \bullet \\
0 & \bullet \\
0 & \bullet \\
\bullet & \bullet \\
0 & \bullet \\
0 & \bullet \\
\bullet & 0\end{array}\right|$ & $\mid \begin{array}{lll}0 & \bullet \\
0 & \bullet \\
0 & \bullet \\
0 & \bullet \\
0 & \bullet \\
0 & \bullet \\
0 & \bullet \\
0 & \bullet \\
0 & \bullet \\
0 & \bullet \\
0 & \bullet & 0\end{array}$ & $\left|\begin{array}{ccc}0 & \bullet & \bullet \\
0 & \bullet & \bullet \\
0 & \bullet & 0 \\
0 & \bullet & \bullet \\
0 & \bullet & \bullet \\
0 & \bullet & \bullet \\
0 & \bullet & \bullet \\
\bullet & \bullet & \bullet \\
0 & \bullet & \bullet \\
0 & \bullet & \bullet \\
0 & \bullet & \bullet\end{array}\right|$ & $\left|\begin{array}{lll}0 & \bullet & \bullet \\
0 & \bullet & \bullet \\
0 & 0 & \bullet \\
\bullet & 0 & \bullet \\
0 & \bullet & \bullet \\
0 & 0 & \bullet \\
0 & 0 & \bullet \\
0 & \bullet & \bullet \\
0 & \bullet & \bullet \\
\bullet & \bullet & \bullet \\
\bullet & \bullet & \bullet\end{array}\right|$ & $\left|\begin{array}{ll}0 & \bullet \\
0 & \bullet \\
0 & \bullet \\
0 & \bullet \\
0 & \bullet \\
0 & \bullet \\
0 & \bullet \\
0 & \bullet \\
0 & \bullet \\
0 & \bullet \\
0 & 0\end{array}\right|$ & $\left|\begin{array}{lll}0 & 0 & 0 \\
0 & 0 & 0 \\
0 & 0 & 0 \\
0 & 0 & 0 \\
0 & 0 & 0 \\
0 & 0 & 0 \\
0 & 0 & 0 \\
0 & \bullet & 0 \\
0 & 0 & 0 \\
0 & 0 & 0 \\
0 & 0 & 0\end{array}\right|$ & 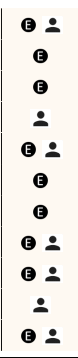 & 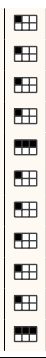 & 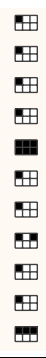 \\
\hline & \begin{tabular}{|l} 
MatrixExplorer \\
NodeTrix \\
Hadlak et al. \\
Overview \\
ConVis \\
TargetVue \\
MediaDiscourse \\
VisOHC \\
iForum \\
VASSL \\
CommAID
\end{tabular} & \begin{tabular}{|l|}
45 \\
46 \\
77 \\
78 \\
79 \\
80 \\
\end{tabular} & $\left|\begin{array}{lllll}\bullet & 0 & 0 & \bullet & 0 \\
\bullet & 0 & 0 & \bullet & 0 \\
0 & 0 & 0 & \bullet & \bullet \\
\bullet & 0 & 0 & 0 & \bullet \\
\bullet & 0 & 0 & \bullet & \bullet \\
\bullet & 0 & 0 & \bullet & 0 \\
\bullet & 0 & 0 & 0 & \bullet \\
\bullet & 0 & 0 & 0 & \bullet \\
\bullet & 0 & 0 & \bullet & 0 \\
\bullet & 0 & 0 & 0 & \bullet \\
\bullet & 0 & 0 & \bullet & \bullet\end{array}\right|$ & $\begin{array}{ll}0 & 0 \\
0 & 0 \\
0 & 0 \\
0 & 0 \\
0 & 0 \\
0 & 0 \\
0 & 0 \\
0 & 0\end{array}$ & $\begin{array}{ll}\bullet & 0 \\
\bullet & 0 \\
\bullet & 0 \\
\bullet & 0 \\
0 & 0 \\
0 & \bullet \\
0 & 0 \\
0 & 0\end{array} \mid$ & $\mid \begin{array}{lll}0 & c \\
0 & c \\
0 & c \\
0 & c \\
0 & c \\
0 & c \\
0 & c \\
0 & c\end{array}$ & $\mid \begin{array}{lll}0 & \\
0 & \\
0 & \\
0 & \\
0 & \\
0 & \\
0 & \\
0 & \\
0 & \\
0 & \end{array}$ & \begin{tabular}{|l|}
$\mathbf{S}$ \\
$\mathrm{S}$ \\
$\mathrm{S}$ \\
$\mathrm{S}$ \\
$\mathrm{S}$ \\
$\mathrm{S}$ \\
$\mathrm{S}$ \\
$\mathrm{S}$
\end{tabular} & $\begin{array}{l}0 \\
0 \\
0 \\
0 \\
0 \\
0\end{array}$ & $\begin{array}{lll}\bullet & \bullet & 0 \\
\bullet & \bullet & 0 \\
\bullet & \bullet & 0 \\
\bullet & \bullet & 0 \\
\bullet & \bullet & 0 \\
\bullet & \bullet & 0 \\
\bullet & \bullet & 0 \\
\bullet & \bullet & 0\end{array}$ & $\left|\begin{array}{lll}\bullet & \bullet & 0 \\
\bullet & \bullet & 0 \\
\bullet & \bullet & 0 \\
\bullet & 0 & \bullet \\
\bullet & \bullet & \bullet \\
\bullet & \bullet & \bullet \\
\bullet & 0 & \bullet \\
\bullet & 0 & \bullet \\
\bullet & \bullet & \bullet \\
\bullet & 0 & \bullet \\
\bullet & \bullet & \bullet\end{array}\right|$ & $\begin{array}{l}\mathbf{S} \\
\mathbf{S} \\
\mathbf{S} \\
\mathbf{S} \\
\mathbf{S} \\
\mathbf{S} \\
\mathbf{S} \\
\mathbf{S}\end{array}$ & $\left|\begin{array}{l}0 \\
0\end{array}\right|$ & $\begin{array}{l}\text { 田 } \\
\text { 困 } \\
\text { 田 }\end{array}$ & $\begin{array}{l}\mathrm{D} \\
\mathrm{D} \\
\mathrm{D} \\
\mathrm{D} \\
\mathrm{D} \\
\mathrm{D} \\
\mathrm{D} \\
\mathrm{D} \\
\mathrm{D} \\
\mathrm{D} \\
\mathrm{D}\end{array}$ & $\left|\begin{array}{ll}\text { II } & \text { I } \\
\text { II } & \text { I } \\
\text { II } & \text { I } \\
\text { IIII II } \\
\text { II } & \text { II } \\
\text { II } & \text { II } \\
\text { II } & \text { I } \\
\text { II } & \text { I } \\
\text { II } & \text { II } \\
\text { II } & \text { I } \\
\text { II } & \text { I }\end{array}\right|$ & $\left|\begin{array}{lll} & 0 & 0 \\
\bullet & 0 & 0 \\
\bullet & 0 & 0 \\
0 & 0 & 0 \\
- & 0 & 0 \\
\bullet & 0 & 0 \\
\bullet & 0 & 0 \\
0 & 0 & 0 \\
- & 0 & 0 \\
\bullet & 0 & 0 \\
0 & 0 & 0\end{array}\right|$ & $\begin{array}{l}\bullet \\
\bullet \\
\bullet \\
\bullet \\
\bullet \\
\bullet \\
\bullet \\
\bullet\end{array}$ & $\left|\begin{array}{ll}\bullet & \bullet \\
\bullet & \bullet \\
0 & \bullet \\
\bullet & \bullet \\
\bullet & \bullet \\
\bullet & \bullet \\
\bullet & \bullet \\
\bullet & \bullet\end{array}\right|$ & $\mid \begin{array}{lll}0 & \bullet \\
0 & \bullet \\
0 & \bullet \\
0 & \bullet \\
0 & \bullet \\
0 & \bullet \\
0 & \bullet \\
0 & \bullet \\
0 & \bullet \\
0 & \bullet \\
0 & \bullet & 0\end{array}$ & $\left|\begin{array}{ccc}0 & \bullet & \bullet \\
0 & \bullet & \bullet \\
0 & \bullet & \bullet \\
0 & \bullet & \bullet \\
0 & \bullet & \bullet \\
0 & \bullet & \bullet \\
0 & \bullet & \bullet \\
0 & \bullet & \bullet \\
0 & \bullet & \bullet \\
0 & \bullet & \bullet \\
\bullet & \bullet & \bullet\end{array}\right|$ & $\left|\begin{array}{lll}\bullet & 0 & \bullet \\
\bullet & 0 & \bullet \\
\bullet & 0 & \bullet \\
0 & \bullet & \bullet \\
\bullet & \bullet & \bullet \\
\bullet & \bullet & \bullet \\
\bullet & \bullet & \bullet \\
\bullet & \bullet & \bullet \\
\bullet & \bullet & \bullet \\
\bullet & 0 & \bullet \\
\bullet & 0 & \bullet\end{array}\right|$ & $\left|\begin{array}{ll}0 & \bullet \\
0 & \bullet \\
0 & \bullet \\
0 & \bullet \\
0 & \bullet \\
0 & \bullet \\
0 & \bullet \\
0 & \bullet \\
0 & \bullet \\
0 & \bullet \\
0 & 0\end{array}\right|$ & $\left|\begin{array}{lll}0 & 0 & 0 \\
0 & 0 & 0 \\
0 & 0 & 0 \\
0 & 0 & 0 \\
0 & 0 & 0 \\
0 & 0 & 0 \\
0 & 0 & 0 \\
0 & 0 & 0 \\
0 & 0 & 0 \\
0 & 0 & 0 \\
0 & 0 & 0\end{array}\right|$ & 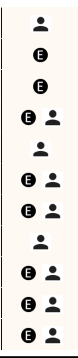 & 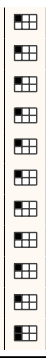 & 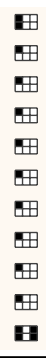 \\
\hline & $\begin{array}{l}\text { Zhao et al. } \\
\text { Whisper } \\
\text { ConToVi } \\
\text { Beagle }\end{array}$ & \begin{tabular}{|l|}
85 \\
86 \\
87 \\
\end{tabular} & $\left|\begin{array}{lllll}- & 0 & 0 & 0 & 0 \\
- & 0 & 0 & \bullet & \bullet \\
- & 0 & 0 & 0 & 0 \\
- & 0 & 0 & \bullet & 0\end{array}\right|$ & $\left|\begin{array}{ll}\bullet & 0 \\
\bullet & 0 \\
0 & 0 \\
0 & 0\end{array}\right|$ & $\begin{array}{ll}\bullet & 0 \\
\bullet & 0\end{array}$ & $\mid \begin{array}{l}\bullet \\
0\end{array}$ & \begin{tabular}{l|l}
0 \\
0
\end{tabular} & $\mathbf{S}$ & $\begin{array}{l}\mathbf{0} \\
\mathbf{0}\end{array}$ & $\begin{array}{ll}0 & 0 \\
0 & 0\end{array}$ & $\left|\begin{array}{lll}\bullet & 0 & \bullet \\
\bullet & \bullet & \bullet \\
0 & 0 & \bullet \\
\bullet & \bullet & \bullet\end{array}\right|$ & \begin{tabular}{|l}
$\mathbf{S}$ \\
$\mathbf{S}$ \\
$\mathbf{S}$
\end{tabular} & 0 & 田 & $\begin{array}{l}\mathrm{D} \\
\mathrm{D} \\
\mathrm{D} \\
\mathrm{D}\end{array}$ & $\left|\begin{array}{cc}\text { II } & \text { I } \\
\text { IIII } & \text { I } \\
\text { IIII } & \text { II } \\
\text { IIII } & \text { II }\end{array}\right|$ & $\left|\begin{array}{lll}- & 0 & 0 \\
\bullet & 0 & 0 \\
- & 0 & 0 \\
- & 0 & 0\end{array}\right|$ & $\mid \begin{array}{llllll}\bullet & \bullet & \bullet & \bullet & \bullet & \bullet \\
\bullet & \bullet & \bullet & 0 & \bullet & \bullet \\
\bullet & \bullet & \bullet & 0 & \bullet & \bullet\end{array}$ & $\left|\begin{array}{ll}\bullet & \bullet \\
\bullet & \bullet \\
\bullet & \bullet\end{array}\right|$ & $\left|\begin{array}{ll}0 & \bullet \\
0 & \bullet \\
0 & \bullet\end{array}\right|$ & $\left|\begin{array}{lll}0 & \bullet & \bullet \\
0 & \bullet & \bullet \\
0 & \bullet & \bullet \\
0 & \bullet & \bullet\end{array}\right|$ & $\left|\begin{array}{lll}0 & \bullet & \bullet \\
\bullet & \bullet & \bullet \\
0 & \bullet & \bullet \\
\bullet & \bullet & \bullet\end{array}\right|$ & $\left|\begin{array}{ll}0 & \bullet \\
0 & \bullet \\
0 & \bullet \\
0 & \bullet\end{array}\right|$ & $\left|\begin{array}{lll}\bullet & 0 & 0 \\
0 & 0 & 0 \\
0 & 0 & 0 \\
0 & 0 & 0\end{array}\right|$ & $\begin{array}{l}\theta \div \\
\theta \div \\
\theta \div\end{array}$ & $\begin{array}{l}\mathbb{⿴ 囗 十} \\
\mathbb{1} \\
\mathbb{⿴ 囗 十}\end{array}$ & $\begin{array}{l}\boxplus \\
\boxplus \\
\boxplus\end{array}$ \\
\hline
\end{tabular}


(e.g., [40]), encompassing the content and the communication network, respectively. However, the other data types are also relevant. For example, communication can happen via audio (e.g., telephone or VoIP) or via video chats, comprising audio and moving images, i.e., video data. While our framework focuses primarily on this written (i.e., text) communication, it is agnostic to the data type of information. For example, it would be possible to include the detection of facial expressions using deep learning [89] to analyze the analogical code and set it in context. The last data type is time-series data (e.g., [47]), often counted to the meta-data and can, for example, contain information about communication regularity.

Code/Intent - The meaning is the central aspect of the communication, as evident by the plethora of approaches that aim to learn it. Based on Watzlawick et al. [11], the communication can be regarded as code, either digital or analogical (sic!). In a simplified expression, the digital code refers to the actual meaning of the transmitted information in a symbolic system, while the analogical code refers to how something is communicated, including cues (e.g. biosignals), and context information. While the former is, by definition, present in virtually all approaches we compared (e.g., [22], [45]), analogical analysis is rare (e.g., [79]).

Expressiveness - Similar, but conceptually slightly different, is the expressiveness, which describes the nature of the information extracted from the content. It can be explicit factual information that is evident directly from the content or information implicitly contained and must be inferred, for example from the semantic. Code and expressiveness together allow to classify systems by their capability to leverage both digital (e.g., actual content information) and also analogical codes (e.g., sentiment analysis) while judging support for explicit (e.g., keyword-based search like [44]) and also implicit (e.g., named entity recognition like [63] ) content. Most approaches consider the explicit level, and several, especially text-based ones, also consider the implicit level.

\subsubsection{Communication Participants}

A second, very important aspect in communication research, is the relationship of the communication participants. It is usually analyzed both from the perspective of scope or by their power relationship.

Target Scope - The target scope can be oneself (e.g., in a note to oneself), a single person, or a group. The analysis of the existing approaches shows that most systems focus on a single target.

Power Relationship - It comes again from classical communication research [11], which proposes a distinction into symmetrical (equal grounds) or complementary (dependence). The relationship context is rarely taken into account explicitly in existing research (e.g., [86]).

\subsubsection{Environment}

The context of communication is very important [11], because it can influence the exchange itself, such that message properties have to be interpreted differently.

Collection Type - The collection type defines how the data was acquired and how it is leveraged. We propose to categorize it into a quadrant, consisting of the targeting methodology on the one side and the anonymity level on the other side (see the relevant part in Figure 3). The former can be either targeted (specific communication from a restricted set of users due to a trigger event) or untargeted (unfound bulk collection). The latter can either be high (anonymized or pseudo-anonymized) or low (identifiable).
Regarding the analysis, this can make a significant difference. For example, the targeted analysis of identifiable communication participants can focus on the actual exchange and leverage context and relationship information. The untargeted analysis of pseudoanonymized communication instead often results in a search for the needle in the hay-stack and can not always leverage background information. This results in vastly different application development, scalability, and inference capabilities.

Analysis Coupling - Closely related is the analysis coupling, which can influence the communication coding due to privacy considerations. Again, we categorize it into a quadrant, this time between (expected) knowledge about the analysis, which can be either known or unknown to be true. Note that the inverse, known to be false, is only achievable with a specific degree of certainty. The coupling refers to the effect that the (expected) analysis can influence the measurement. If participants expect their communication to be analyzed, they might communicate differently, being less honest, implicit, or communicate not at all. This effect also depends on the targeting: If it is known that all e-mails in the world are collected and analyzed (untargeted), they might have less influence than knowing that their e-mails are monitored for sure (targeted), as this can influence the degree of scrutiny and sophistication of the analysis employed. For example, diplomatic cables are often very frank, mainly because they are not expected to be leaked (assume untargeted, identifiable), while public policy discussions are much more considerate (targeted, identifiable).

Confidentiality - The previous point relates to the confidentiality of the communication channel, which can influence the communication coding, and therefore should be taken into account.

Strategy - A technical consideration which influences the analysis possibilities is the collection strategy. Is the data leveraged just a one-off dataset that contains past information only (static $\mathbf{S}$ ) like [39], or does the data collection reach the present, possibly is actively running, and the information increases over time (dynamic D ) like [24]. They differ as the extracted knowledge might (significantly) change over time with new information available, affecting the analytical process.

Continuity - Along with the previous consideration, one can consider the technical realization of a dynamic data collection. The options are either a one time only $\mathbf{O}$ approach, where a fixed dataset is loaded once (e.g., [83]), a batch $\mathbf{B}$ approach that supports an extension with a new data (e.g., [44|) or, a streaming $\mathbf{S}$ approach for a continuous integration (e.g., [86]). However, most approaches only support $\mathbf{O}$.

\subsection{Processing and Models}

This category focuses on the particularities of the processing and the models, mainly from a technical perspective in visual interactive data analysis, in the three subcategories, namely the analytical goal (why?), the model scope (what?) and the processing (how?).

\subsubsection{Analytical Goal}

One essential distinguishing criteria between approaches are the supported methodologies to achieve the analytical goal. We align this classification on the standard definition of analytical tasks in visual analytics [90]. This includes the category representation for a fixed analysis task, confirmatory analysis for a directed search and exploratory analysis for a undirected search (supported by most approaches). Another goal in communication analysis involves predictive analysis (e.g., [21]), which could also be regarded as a sub-goal of all methodologies. 


\subsubsection{Model Scope}

Here, we discuss generic capabilities of the analysis models.

Modality - The analysis modality refers to the employed concepts and categorizes into the three common aspects: meta-data (e.g., [47]), network (e.g., [40|), and content (e.g., [73]), as used in many technical descriptions of communication analysis.

Collection - The different approaches provide various levels of support for multiple collection environments. For example, many systems can only consider a single dataset (e.g., a set of e-mails) and therefore support only single-environment $\mathbf{S}$ (e.g., [61]), while a few also support the integration of multiple data sources, which makes them multi-environment $\mathbf{M}$ systems (e.g., [44]).

Data-Mapping - While most systems require a fixed data format, some also support a flexible import system which allows to map properties and, in case they support multiple environments, to correlate different aspects, for example sender and usernames.

\subsubsection{Processing}

Due to the breadth of different methods, we focus on generic aspects, namely the support for group communication analysis, and considerations for latency and scalability.

Group Communication - One rather interesting aspect we discovered during the analysis of the approaches is the varying support of group communications. Practically all approaches support an individual to individual analysis. We propose to structure the approaches based on their support to analyze the communication between a source and a target in a $3 \times 3$ matrix, e.g., individual to individual is encoded as 进 (e.g., [80]). These entities can be either individuals, groups, or groups within groups. The difference between the two latter is subtle but involves the support of boundary restrictions on sub-groups instead of overlapping group assignments. One might expect the matrix to be symmetric, but this might not be always the case.

Latency - Research [12] indicates that latency in the communication can significantly affect it. The two primary options are (nearly) instantaneous communication, like in an active live $\mathbf{L}$ chat (e.g., [24]) or delayed $\mathbf{D}$ communication (e.g., [82]), such as e-mail or as a document. Note that the choice is not purely categorical, and from a technical perspective, this could also be considered a numerical property. Differentiation into these two groups [12] is often enough for most differences in reaction and behavior, although the latency can, of course, play a role (e.g., answering under time pressure). In consideration of analogical codes, however, it makes sense to include a third, orthogonal category: In-person I communication (a subgroup of live communication) can exhibit markedly different results compared to live chat conversations.

Scalability - The scalability can be defined on two levels: First, on the data-ingress level, which defines the amount of input data the system can import, analyze, and visualize initially. The second, more subtle aspect is the scalability on the search and analysis side, for example, during exploratory analysis. For example, how many results can be shown simultaneously? We roughly categorize both aspects into few (less than ten, I), medium (order of hundredths-thousands, II), and huge (more than 10k, IIII).

\subsection{Visual Interface 囵}

Here, we describe the visual interface on an abstract level.

\subsubsection{Representation}

The central aspect of visualization systems are its visualizations.

Method - We follow the established nomenclature of visualization techniques for the visualization method. However, we only chose the three most common ones, which account for the vast majority of approaches, comping up with the following selection: node-link-based (e.g., [39]), timeline-based (e.g., [70]), and matrix-based (e.g., [67]). Alternatively, there is an option for other (e.g., [87]) techniques and multiple (e.g., [83]) for multiparadigm approaches.

Pane - The different visualization methods can be employed in different visualization panes. We consider the three major ones, namely 2D, 3D, and VR. For example, a communication network can be visualized as a node-link diagram in either way, and each choice may influence the interaction concepts.

\subsubsection{Interaction}

Interaction methods are of central importance in visual analytics.

Operation Method - We classify the approaches based on their interaction method according to the classification developed by Yi et al. [91], namely Select, Explore, Reconfigure, Encode, Abstract/Elaborate, Filter, and Connect. Some are extremely common, while others like encode depend on the capabilities.

Manipulation - The manipulation of the elements can be either direct, for example when interacting with data or visual objects. Alternatively, it can be indirect, for example when modifying parameters. Most approaches support both.

\subsubsection{Refinement}

In addition to the interaction concept, other discerning factors are the particularities of the refinement. For these concepts, we also align with standard visual analytics concepts.

Goal - Two primary goals can be differentiated: is the goal to tune an underlying model (e.g., [67]) or the data (e.g., [72])?

Strategy - The strategy might vary greatly with the approach: does it follow an active learning approach (e.g., [67]), or is the process either iterative (e.g., [47]) or progressive (e.g., [87]) instead?

\subsection{Knowledge Generation}

The last category concerns the particularities of the knowledge that can be generated and learned. We propose the three subcategories output, verification approach, and knowledge gain.

\subsubsection{Output}

Based on the classification of Spinner et al. [92], we propose two distinct categories for the learned knowledge type: An explanation can consist, for example, of numerical (e.g., [40]), textual (e.g., [75]), or graphical representations (e.g., [65]). It represents knowledge but in a factual representation that is not easily transferable and can be regarded as a (final) result of the existing data. In contrast to this, another type of result can be a transition function, which is closer to an actual model, one example being the analysts mental model. Another type is a machine model (e.g., [21], for example, a trained, applicable classifier that encapsulates learned knowledge. 


\subsubsection{Verification Approach}

The presentation of knowledge raises the question of its verification.

Factors - Visual analytics is very well suited to address important factors like confidence, trust and privacy. For example, probability scores could be used to estimate the results' confidence stemming from automatic processes (e.g., [67]) and visualize it to the expert. Other examples include analysis logfiles, integrity protection, traceability, and verify-ability, as well as a provenance history. Lack of such certainty measures might exclude systems from sensitive areas. While essential, many approaches do not consider this as much as would be desirable.

Evaluation - To evaluate approaches, the authors own evaluation method can be considered. Several options are possible: Either a convincing example $\mathbf{E}$ or a case study (e.g., [46]). A second option is a comparison $\leftrightarrow$ with similar, existing approaches (e.g., [67]). A third option would be a qualitative interview (e.g., [8]) or a quantitative user study $\bullet$.

\subsubsection{Knowledge Gain}

As a final step in the learning process, the question arises which knowledge is actually gained and how powerful the process is.

Time Dimensionality - The time dimensionality describes the relationship between data and knowledge generation. A $2 \times 3$ matrix shows the possible combinations of data basis and prediction type, each with the entries past, present, and future. For example $\boxplus$ (e.g., [77]), a system can use past data and predict past data, for example for a search. Then, $-($ e.g., [76]) would be an article analysis and prediction system which has been trained on past data to analyze a text, either an existing one or one on the fly in the present and future. Another example $\boxplus$ for a future prediction is a model that forecasts communication activity based on past events. Note that the future cannot be the basis due to causality.

Predictive Power - A second important consideration describes the predictive power of the knowledge generated, which is represented as a $2 \times 3$ matrix, where the result (explanation or transition function) and the time are combined. For example $\boxplus$, can a system explain (i.e. show) past events (virtually all systems)? Or is it able $=$ to provide factual information for future events (e.g., [76], internal model is inaccessible). A more powerful example $\mathbf{m}$ is a controllable model which can explain and predict (e.g., [21]).

Domain-specific Aspects - Last, but not least, depending on different analysis tasks, more specific options might be of interest. As discussed above, these are out of scope here, however, we could imagine this as future work (see Section 5 .

\section{Discussion ANd FutURe Work}

This section discusses the main findings and lessons learned before reflecting on the difficulties in creating a conceptual framework for communication analysis. In particular, we discuss the potential implications and opportunities for future research while highlighting the shortcomings in the current formalization.

We have defined four main dimensions containing 30 different properties, providing a conceptual framework for interactive communication analysis systems employing visual analytics principles.

We imagine several applications for this framework: Its primary goal is to structure the research field and provide a common language for the community. Simultaneously, it can aid researchers in identifying research gaps, thereby supporting the transfer of theoretical concepts into practical systems. Further, it allows practitioners to chose the best fitting approach depending on their needs or checking out similar approaches, while enabling developers to judge the domain cover and maturity of their systems. Lastly, it can form the foundation for a more detailed survey in the field of automated communication analysis.

\subsection{Survey Findings and Research Opportunities}

Visual analytics is especially suited to support semi-automatic communication analysis [26], [28]. The complexity, multi-modality, and ambiguity of communication make it an ideal companion to interactively combine domain knowledge from experts and computing power. Concepts like interactive learning allows to refine models, while uncertainty awareness enables judging automatic results, fostering user trust and possibly identifying bias.

Findings - To apply the framework, we have taken the 41 selected approaches (see Section 3 and coded them according to our conceptual framework. Based on the results in Table 2, we can discover several interesting aspects. For once, for the data type, while the analysis of text data seems mostly universal across representation methods, this is not the case for the other data types. Somewhat unsurprisingly, when network data is included, the visualization is often node-link-based or multi-paradigm, while for time-series, it is either timeline or multi-paradigm. Given the scope of the survey, the lack of audio and image is not surprising. Given that all the approaches belong to the category of visual analytics, it is also unsurprising that virtually all support representative, confirmatory and exploratory analysis as their analysis methodology, their operation methods covers most options, and their explanation is at least always graphical.

More interesting, however, are the differences and the research opportunities we can conclude from their discrepancies, which we highlight in the following for each category (see Figure 1); those of particular relevance are highlighted with an star: $\star$.

\section{I.1 Analysis of the Meaning and Analogical Code}

Only a subset of approaches analyzes the implicit meaning of the communication (e.g., [63], [79], [83]). However, almost none analyze the analogical code of the communication.

Implication: The analogical code can contain important cues with might support the analysis of the content and provide supportive information about the relationships inside the network, which makes it especially relevant to consider. Leveraging it can lead to a richer and more-complete analysis, while it can support resolving contradictions and ambiguities.

\section{I.2 Include Power Relations}

Again, almost no approach considers the power relations between the participants, which can similarly influence the communication semantics, meaning, and modalities.

Implication: Power relations between participants might influence content aspects like choice of words, formality, use of irony, meaning, or meta-data aspects like the speed / timestamps / amount of messages. Results can be used and considered in context with the content analysis.

\section{I.3 Dynamic Analysis}

While some might consider this a production problem, the development of systems that support the dynamic analysis of communication data and batch/stream approaches also sets considerable hurdles to established analysis and visualization methods, which makes it an interesting academic research 
problem.

Implication: Exploring how new data and updated results can be integrated, how fluctuating analysis can be stabilized, and how changed predictions are communicated offers more effective ways for visual communication.

\section{I.4 Research the Analysis Coupling}

There is practically no research on how a system can mitigate a possible analysis coupling.

Implication: Being aware of the measurement problem and explore mitigation can strengthen user confidence and trust, while avoiding missed or erroneous results.

\section{II.1 Multi-Environment Inclusion}

Many approaches can only handle a single data source (environment) and offer no data mapping (like, e.g., [44]). Instead, these steps are outsourced to preprocessing. Their inclusion could make leveraging multiple data sources simultaneously less complicated.

Implication: Automating the merging of heterogeneous data sources with few or no user input reduces the amount of manual preprocessing or knowledge transfer required, while exploring optimal interface strategies.

\section{II.2 Analyze Group Communication}

Only a few approaches support the analysis of group communication (e.g., [87]), and almost none support nested groups. However, much communication actually happens inside or between groups, which can involve specific particularities.

Implication: New and more detailed knowledge can be drawn how groups operate and information diffuses within them.

\section{III.3 Explore Visualization Panes}

Practically all approaches use the 2D pane for visualizations. It might be worthwhile to explore 3D and VR concepts. Especially, for example, for network analysis, VR might offer benefits.

Implication: 3D or VR might provide benefits for communication analysis specific tasks in particular, improving the analysis in different levels.

\section{IV.1 Model / Transfer Function / Knowledge Gain}

Few approaches contain an actual, powerful machine model to analyze communication (e.g., [23], [67]).

Implication: Using such models can potentially support the analysis, through measures as active learning, intelligent filtering, or confidence-based predictions. Transfer Functions allow for a more universal machine learning, applying knowledge to new problems, increasing the predictive power. This reduces manual work and increases the analytical capabilities.

\section{IV.2 Confidence, Trust, and Privacy}

These factors are insufficiently considered the majority of approaches, leading to a black-box analysis. Instead, one could include confidence estimates (e.g., [67]), logs, provenance (e.g., [8]), data minimization, or other concepts. Implication: Several applications have strong requirements for confidence, trust, provenance, or privacy. Exploring how these can be fulfilled without limiting the analysis can replace manual analysis by automated system.

\section{IV.3 Comparisons and Quantitative User Studies}

While many approaches include case studies and (qualitative) expert interviews, almost none do actual comparisons with related approaches or conduct quantitative user studies.

Implication: Case studies and qualitative expert interview are not always comparable or conducted to the same standards. For reliable comparisons between approaches detailed comparisons or quantitative studies are required, which provide a more objective overview of the field.

\section{O.1 Holistic Approaches}

Only a few approaches work towards a holistic analysis by considering multiple analysis aspects together and in context, covering all modalities.

Implication: A holistic perspective can increase the analytical capabilities considerably, supporting cross-matches beyond analysis boundaries, while reducing mental load and manual work required.

\section{O.2 Context / Analysis Reference Window}

Similar to the holistic analysis, a specific focus on the communication context in reference to each other should be explored further for both inter- and intra-modality analysis.

Implication: Few approaches consider other modalities or external factors to explain particularities. For example, a break in a communication sequence might appear as a gap, but when combined with location information (e.g., same building) might indicate that the participants might have met for lunch and continued their conversation there, indicate an air travel period, etc. In summary, the correct interpretation of communication is extremely context depended. This context, however, is variable and many different ones exist, which is why knowing the relevant ones improves the accuracy, also of the dependent methods.

Implications - All the previously described opportunities offer potential improvements for a more complete analysis of communication. Fusing together multiple methods can lead to a richer and more complete analysis, potentially resolving contradictions and ambiguities. Some opportunities might primarily support existing analysis steps (e.g., I.2) as a precursor, while others provide new areas in itself (e.g., II.2, O.1). While not all discussed aspects might always be relevant in any given analysis, our framework identifies and describes areas that users can consider and potentially leverage, depending on their analytical needs.

\subsection{Limitations and Future Work}

While our conceptual framework offers exciting research opportunities for more powerful communication analysis systems and the application of visual analytics concepts, the area itself is under active research with several limitations and unsolved challenges.

One problem with the described taxonomy is the basis it is designed upon (completeness). We are confident we included the essential results from classical communication research, technical considerations, and many aspects from the surveyed approaches. However, one issue is the completeness of the surveyed approaches. A significant problem in this research area is that relevant approaches are rarely labeled as belonging to communication analysis and are not easily definable by a set of keywords. We initially thought about compiling a list of domain-specific keywords to select papers by, for example, social network analysis, sentiment 
analysis, e-mail analysis, etc. However, we found it highly likely that such a selection would be highly biased by our knowledge of relevant topics, which is why we decided to go for a more extensive drag-net search to include previously unknown areas. However, while we took great care when defining the seed search and then selecting the approaches, it is inadvertently likely we missed individual ones, not least by restricting the target journals and conferences. Also, it could happen that a few approaches fell through our automatic or manual search pattern (see Section 3), while still being applicable to communication analysis. Some missing approaches may contain novel aspects which might widen the framework and introduce new elements or pose problems for existing ones. However, as this paper is not a complete survey and the collection of approaches is primarily used as one of three parts to design a helpful formalization scheme, the collection only has to cover most techniques and different methods and still be beneficial for many approaches. Nevertheless, to address the issue of missing approaches, we created an accompanying survey-like website available at communication-analysis.dbvis.de, which lists the approaches we considered and also allows readers to submit methods they consider relevant and that have not yet been included.

While the previous limitations were concerned with the selected approaches, another possible limitation concerns our framework itself. It may contain overlaps, and the basis it provides is not entirely orthogonal. Given the complexity of the topic, we think it is challenging to create a wholly consistent yet easily usable taxonomy, and there is a need to balance the different trade-offs. The choices we made for selecting the categories are based on existing research and approaches and justified when required. However, given sparse taxonomy and non-standardized vocabulary, some groupings and namings could arguably have been chosen differently with the same validity. To advance research in this area, we decided to propose our framework as a first possible draft and one step towards a universally accepted framework. We, therefore, invite the research community to give feedback to stimulate the scientific discussion and extend upon the framework in further iterations, which can be enhanced further by more input from diverse research communities. As part of this process, the individual, multi-faceted aspects can be formalized in more detail.

Another aspect is the extension of the framework to nonhuman communication. Several aspects of the framework could similarity be applied to communication in general, for example, machine to machine. Indeed nothing in the framework is specifically tailored to a human communicator, as all the properties could also apply to other types of communication. However, human communication is often more nuanced than machine communication, making parts of the framework less relevant, while other features (e.g., structuring, exchange content scope) might be missing so far.

\section{Conclusion}

In the last decades, communication analysis has experienced a shift away from manual analysis to computer-aided or even highly automated approaches. However, to the same extend as automation levels increased, the analysis itself has often become more specialized, moving away from an overarching exploration. This trend is in contradiction to traditional communication research, which stresses the importance of an holistic approach to capture the full meaning and context of communication. As a result, many modern digital communication analysis systems are highly adapted to a narrow range of tasks, often either in the area of content or in network analysis. While this might be perfectly sufficient and suitable for their intended use, such an isolated analysis can sometimes lead to an less effective exploration and lead to incomplete or biased results. Using separate approaches requires more manual work, often complicates analysis tasks, can introduce domain discontinuities, and increase the struggle domain experts face when trying to integrate their domain knowledge. Further, isolated analysis may not be sufficient to capture the full available information and can make the automatic as well as the manual detection of cross-matches more difficult. The development of more holistic and advanced approaches for automated communication analysis systems is hindered by the lack of a clear framework and the absence of a common language which combines both technical aspects as well as results from traditional communication research.

We address this challenge by developing and formalizing a design space for digital communication analysis systems based on the existing tool landscape and communication research, while making the case how visual analytics principles can be employed for a more holistic approach. By systematically discussing and structuring the different analysis areas and aspects of the design space we arrive at an conceptual framework to provide an overview and assess the maturity of communication analysis systems. As part of an initial survey, we have also categorized a large set of existing approaches using our framework.

By bridging the gap in the formalization of digital communication analysis systems by describing a design space for communication analysis, we aim to provide researchers with a common language, provide guidelines for building and assessing the maturity of such approaches, as well as point out gaps in the literature which offer exciting research opportunities. The results of this work are widely applicable in a variety of domains which are concerned with communication analysis like civil security, the digital humanities, or business intelligence, both from a theoretical point of view as well as for the development of more powerful communication analysis systems.

\section{ACKNOWLEDGMENTS}

The authors acknowledge the financial support by the Federal Ministry of Education and Research of Germany (BMBF) in the framework of PEGASUS under the program "Forschung für die zivile Sicherheit 2018 - 2023" and its announcement "Zivile Sicherheit - Schutz vor organisierter Kriminalität II".

\section{References}

[1] D. A. Keim, G. Andrienko, J.-D. Fekete, C. Görg, J. Kohlhammer, and G. Melançon, "Visual Analytics: Definition, Process, and Challenges," in Information Visualization, ser. Lecture Notes in Computer Science, A. Kerren, Ed. Springer, 2008, vol. 4950, pp. 154-175.

[2] N. M. Su and G. Mark, "Communication chains and multitasking," in Proceeding of the twenty-sixth annual CHI conference on Human factors in computing systems - CHI '08, M. Czerwinski, A. Lund, and D. Tan, Eds. New York, New York, USA: ACM Press, 2008, p. 83.

[3] M. H. Nguyen, J. Gruber, J. Fuchs, W. Marler, A. Hunsaker, and E. Hargittai, "Changes in Digital Communication During the COVID-19 Global Pandemic: Implications for Digital Inequality and Future Research," Social Media + Society, vol. 6, no. 3, p. 205630512094825, 2020.

[4] C. A. Scolari, "Mapping Conversations About New Media: The Theoretical Field of Digital Communication," New Media \& Society, vol. 11, no. 6, pp. 943-964, 2009.

[5] M. Trier, "Research Note - Towards Dynamic Visualization for Understanding Evolution of Digital Communication Networks," Information Systems Research, vol. 19, no. 3, pp. 335-350, 2008. 
[6] S. C. Herring, "The Coevolution of Computer-Mediated Communication and Computer-Mediated Discourse Analysis," in Analyzing Digital Discourse, P. Bou-Franch and P. Garcés-Conejos Blitvich, Eds. Springer, 2019, pp. 25-67.

[7] P. W. Foltz and M. J. Martin, "Automated Communication Analysis of Teams," Team Effectiveness in Complex Organizations, 2008.

[8] M. T. Fischer, D. Seebacher, R. Sevastjanova, D. A. Keim, and M. ElAssady, "CommAID: Visual Analytics for Communication Analysis through Interactive Dynamics Modeling," Computer Graphics Forum, 2021.

[9] G. Simmel, Soziologie: Untersuchungen über die Formen der Vergesellschaftung. Leipzig: Duncker \& Humblot, 1908.

[10] J. L. Moreno, Who Shall Survive? A New Approach to the Problem of Human Interrelations, Washington, D.C., 1934.

[11] P. Watzlawick, J. H. Beavin, and D. D. J. Jackson, Menschliche Kommunikation: Formen, Störungen, Paradoxien, 4th ed. Hans Huber, 1974.

[12] J. C. Pearson, Human Communication, 4th ed. New York: McGraw-Hill, 2011.

[13] G. S. Mesch, "Social Context and Communication Channels Choice Among Adolescents," Computers in Human Behavior, vol. 25, no. 1, pp. 244-251, 2009.

[14] I. R. Savage and K. W. Deutsch, "A Statistical Model of the Gross Analysis of Transaction Flows," Econometrica, vol. 28, no. 3, pp 551-572, 1960. [Online]. Available: http://www.jstor.org/stable/1910131

[15] W. van Atteveldt and T.-Q. Peng, "When Communication Meets Computation: Opportunities, Challenges, and Pitfalls in Computational Communication Science," Communication Methods and Measures, vol. 12 no. 2-3, pp. 81-92, 2018.

[16] S. Flensburg and S. S. Lai, "Mapping Digital Communication Systems: Infrastructures, Markets, and Policies as Regulatory Forces," Media, Culture \& Society, vol. 42, no. 5, pp. 692-710, 2020.

[17] I. Onyeator and N. Okpara, "Human Communication in a Digital Age: Perspectives on Interpersonal communication in the family," New Media and Mass Communication, vol. 78, pp. 35-45, 2019.

[18] C. D. Manning and H. Schütze, Foundations of Statistical Natural Language Processing. Cambridge, Mass. and London: MIT Press, 1999.

[19] J. Scott, Social Network Analysis, 4th ed. Los Angeles: SAGE, 2017.

[20] R. Hadjidj, M. Debbabi, H. Lounis, F. Iqbal, A. Szporer, and D. Benredjem, "Towards an Integrated E-mail Forensic Analysis Framework," Digital Investigation, vol. 5, no. 3-4, pp. 124-137, 2009.

[21] Y. Wu, S. Liu, K. Yan, M. Liu, and F. Wu, "OpinionFlow: Visual Analysis of Opinion Diffusion on Social Media," IEEE Transactions on Visualization and Computer Graphics, 2014.

[22] J. Koven, C. Felix, H. Siadati, M. Jakobsson, and E. Bertini, "Lessons Learned Developing a Visual Analytics Solution for Investigative Analysis of Scamming Activities," IEEE Transactions on Visualization and Computer Graphics, vol. 25, no. 1, pp. 225-234, 2019.

[23] Nuix Pty Ltd, "Nuix Discover and Nuix Investigate," 2020. [Online]. Available: https://www.nuix.com/products

[24] Palantir Technologies, Inc., "Gotham," 2020. [Online]. Available: https://www.palantir.com/palantir-gotham/

[25] DataWalk Inc., "DataWalk," 2020. [Online]. Available: https: //datawalk.com/

[26] S. C. Herring, "New Frontiers in Interactive Multimodal Communication," The Routledge Handbook of Language and Digital Communication, pp. 398-402, 2015.

[27] D. Sacha, A. Stoffel, F. Stoffel, B. C. Kwon, G. P. Ellis, and D. A. Keim, "Knowledge Generation Model for Visual Analytics," IEEE Transactions on Visualization and Computer Graphics, vol. 20, no. 12, pp. 1604-1613, 2014.

[28] C. Jensen, S. D. Farnham, S. M. Drucker, and P. Kollock, "The Effect of Communication Modality on Cooperation in Online Environments," in Proceedings of the SIGCHI conference on Human factors in computing systems - CHI 'OO, T. Turner and G. Szwillus, Eds. New York, New York, USA: ACM Press, 2000, pp. 470-477.

[29] C. H. Cooley, Social Organization: A study of the Larger Mind. New York: Charles Scribner's Sons, 1909.

[30] W. Lippmann, Public Opinion. London: G. Allen \& Unwin, 1922.

[31] A. Bavelas, "Communication Patterns in Task-oriented Groups," The Journal of the Acoustical Society of America, vol. 22, no. 6, pp. 725-730, 1950.

[32] H. J. Leavitt, "Some Effects of Certain Communication Patterns on Group Performance," The Journal of Abnormal and Social Psychology, vol. 46, no. 1, p. $38,1951$.
[33] F. Schulz von Thun, Miteinander reden: Störungen und Klärungen - Psychologie der zwischenmenschlichen Kommunikation, ser. Rororo. Reinbek, Hamburg: Rowohlt, 1981.

[34] E. M. Rogers and D. L. Kincaid, Communication Networks: Toward a New Paradigm for Research. Macmillan USA, 1980.

[35] G. Gerbner, "Analysis of Communication Content," 1969.

[36] M. Lombard, J. Snyder-Duch, and C. C. Bracken, "Content Analysis in Mass Communication: Assessment and Reporting of Intercoder Reliability," Human communication research, vol. 28, no. 4, pp. 587604, 2002.

[37] Q. Luo and D. Zhong, "Using Social Network Analysis to Explain Communication Characteristics of Travel-related Electronic Word-ofMouth on Social Networking Sites," Tourism Management, vol. 46, pp. 274-282, 2015. [Online]. Available: https://linkinghub.elsevier.com/ retrieve/pii/S0261517714001393

[38] S. P. Borgatti, A. Mehra, D. J. Brass, and G. Labianca, "Network Analysis in the Social Sciences," Science, vol. 323, no. 5916, pp. 892-895, 2009.

[39] V. Batagelj and A. Mrvar, "Pajek - Program for Large Network Analysis," Connections, vol. 21, no. 2, pp. 47-57, 1998. [Online]. Available: http://mrvar.fdv.uni-lj.si/pajek/

[40] M. Bastian, S. Heymann, and M. Jacomy, "Gephi: An Open Source Software for Exploring and Manipulating Networks," in Proceedings of the International AAAI Conference on Weblogs and Social Media, ser. ICWSM. AAAI, 2009, pp. 361-362. [Online]. Available: http://aaai.org/ocs/index.php/ICWSM/09/paper/view/154

[41] B. Yoon and Y. Park, "A Text-Mining-Based Patent Network: Analytical Tool for High-Technology Trend," The Journal of High Technology Management Research, vol. 15, no. 1, pp. 37-50, 2004.

[42] B. Pang and L. Lee, "Opinion Mining and Sentiment Analysis," Foundations and Trends in Information Retrieval, vol. 2, no. 1-2, pp. 1-135, 2008.

[43] R. Řehůřek and P. Sojka, "Software Framework for Topic Modelling with Large Corpora," in Proceedings of LREC 2010 workshop: New Challenges for NLP Frameworks, Valletta, Malta, 2010, pp. 46-50. [Online]. Available: http://www.fi.muni.cz/usr/sojka/presentations/ lrec2010-poster-rehurek-sojka.pdf

[44] IBM, "i2 Analyst's Notebook," 2020. [Online]. Available: https: //www.ibm.com/us-en/marketplace/analysts-notebook

[45] N. Henry and J.-D. Fekete, "MatrixExplorer: A Dual-Representation System to Explore Social Networks," IEEE Transactions on Visualization and Computer Graphics, vol. 12, no. 5, pp. 677-684, 2006.

[46] N. Henry, J.-D. Fekete, and M. J. McGuffin, "NodeTrix: A Hybrid Visualization of Social Networks," IEEE Transactions on Visualization and Computer Graphics, vol. 13, no. 6, pp. 1302-1309, 2007.

[47] M. Krstajić, E. Bertini, and D. A. Keim, "CloudLines: Compact display of Event Episodes in Multiple Time-series," IEEE Transactions on Visualization and Computer Graphics, vol. 17, no. 12, pp. 2432-2439, 2011.

[48] X. Fu, S.-H. Hong, N. S. Nikolov, X. Shen, Y. Wu, and K. Xu, "Visualization and Analysis of Email Networks," in Proceedings of the 6th International Asia-Pacific Symposium on Visualization, ser. PacificVis. IEEE, 2007, pp. 1-8.

[49] F. Liu, C. Park, Y. J. Tham, T.-Y. Tsai, L. Dabbish, G. Kaufman, and A. Monroy-Hernández, "Significant Otter: Understanding the Role of Biosignals in Communication," in Proceedings of the 2021 CHI Conference on Human Factors in Computing Systems, Y. Kitamura, A. Quigley, K. Isbister, T. Igarashi, P. Bjørn, and S. Drucker, Eds. New York, NY, USA: ACM, 05062021, pp. 1-15.

[50] D. Jagdish, A. Attarwala, and U. Fischer, "Behavior assessment and visualization tool," in CHI '10 Extended Abstracts on Human Factors in Computing Systems, E. Mynatt, D. Schoner, G. Fitzpatrick, S. Hudson, K. Edwards, and T. Rodden, Eds. New York, NY, USA: ACM, 04092010, pp. 3871-3876.

[51] A. Forghani, C. Neustaedter, and T. Schiphorst, "Investigating the communication patterns of distance-separated grandparents and grandchildren," in CHI '13 Extended Abstracts on Human Factors in Computing Systems on - CHI EA '13, W. E. Mackay, S. Brewster, and S. Bødker, Eds. New York, New York, USA: ACM Press, 2013, p. 67.

[52] G. Gao and S. R. Fussell, "A Kaleidoscope of Languages," in Proceedings of the 2017 CHI Conference on Human Factors in Computing Systems, G. Mark, S. Fussell, C. Lampe, m. schraefel, J. P. Hourcade, C. Appert, and D. Wigdor, Eds. New York, NY, USA: ACM, 05022017, pp. 760-772.

[53] A. Mastrianni, L. Kulp, E. Mapelli, and A. Sarcevic, "Understanding Digital Checklist Use Through Team Communication," Extended abstracts on Human factors in computing systems. CHI Conference, vol. 2020, 2020.

[54] J. S. Yi, N. Elmqvist, and S. Lee, "TimeMatrix: Analyzing Temporal Social Networks Using Interactive Matrix-Based Visualizations," International 
Journal of Human-Computer Interaction, vol. 26, no. 11-12, pp. 10311051, 2010.

[55] K. Kucher and A. Kerren, "Text Visualization Techniques: Taxonomy, Visual Survey, and Community Insights," in 2015 IEEE Pacific Visualization Symposium (PacificVis), S. Liu, G. Scheuermann, S. Takahashi, and I. P. V. Symposium, Eds. Piscataway, NJ: IEEE, 2015, pp. 117-121.

[56] E. Hoque and G. Carenini, "ConVisIT: Interactive Topic Modeling for Exploring Asynchronous Online Conversations," in Proceedings of the 20th ACM International Conference on Intelligent User Interfaces, ser. IUI, O. Brdiczka, P. Chau, G. Carenini, S. Pan, and P. O. Kristensson, Eds. New York, USA: ACM, 2015, pp. 169-180.

[57] — - "MultiConVis: Visual Text Analytics System for Exploring a Collection of Online Conversations," in IUI'16, J. Nichols, J. Mahmud, J. O'Donovan, C. Conati, and M. Zancanaro, Eds. New York, USA ACM, 2016, pp. 96-107.

[58] S. Fu, Y. Wang, Y. Yang, Q. Bi, F. Guo, and H. Qu, "VisForum: A Visual Analysis System for Exploring User Groups in Online Forums," ACM Transactions on Interactive Intelligent Systems, vol. 8, no. 1, pp. 1-21, 2018.

[59] J. Zhao, Z. Liu, M. Dontcheva, A. Hertzmann, and A. Wilson, "MatrixWave," in Proceedings of the 33rd CHI Conference on Human Factors in Computing Systems, ser. CHI, B. Begole, J. Kim, K. Inkpen, and W. Woo, Eds. New York, NY, USA: ACM, 2015, pp. 259-268.

[60] A. Perer, I. Guy, E. Uziel, I. Ronen, and M. Jacovi, "The Longitudinal Use of SaNDVis: Visual social Network Analytics in the Enterprise," IEEE Transactions on Visualization and Computer Graphics, vol. 19, no. 7, pp. 1095-1108, 2013.

[61] S. Ghani, B. C. Kwon, S. Lee, J. S. Yi, and N. Elmqvist, "Visual Analytics for Multimodal Social Network Analysis: A Design Study with Social Scientists," IEEE Transactions on Visualization and Computer Graphics, vol. 19, no. 12, pp. 2032-2041, 2013.

[62] S. van den Elzen and J. J. van Wijk, "Multivariate Network Exploration and Presentation: From Detail to Overview via Selections and Aggregations," IEEE Transactions on Visualization and Computer Graphics, vol. 20, no. 12, pp. 2310-2319, 2014.

[63] M. El-Assady, R. Sevastjanova, B. Gipp, D. A. Keim, and C. Collins, "NEREx: Named-Entity Relationship Exploration in Multi-Party Conversations," Computer Graphics Forum, vol. 36, pp. 213-225, 2017 [Online]. Available: https://onlinelibrary.wiley.com/doi/10.1111/cgf.13181

[64] D. Angus, A. Smith, and J. Wiles, "Conceptual Recurrence Plots: Revealing Patterns in Human Discourse," IEEE Transactions on Visualization and Computer Graphics, vol. 18, no. 6, pp. 988-997, 2012.

[65] U. Brandes and B. Nick, "Asymmetric Relations in Longitudinal Social Networks," IEEE Transactions on Visualization and Computer Graphics, vol. 17, no. 12, pp. 2283-2290, 2011.

[66] B. Bach, N. Henry-Riche, T. Dwyer, T. Madhyastha, J.-D. Fekete, and T. Grabowski, "Small MultiPiles: Piling Time to Explore Temporal Patterns in Dynamic Networks," Computer Graphics Forum, vol. 34 no. 3, pp. 31-40, 2015.

[67] M. T. Fischer, D. Arya, D. Streeb, D. Seebacher, D. A. Keim, and M. Worring, "Visual Analytics for Temporal Hypergraph Model Exploration," IEEE Transactions on Visualization and Computer Graphics, vol. 27, no. 2, pp. 550-560, 2020 .

[68] F. B. Viégas, S. Golder, and J. Donath, "Visualizing email content," in Proceedings of the SIGCHI Conference on Human Factors in Computing Systems, R. Grinter, T. Rodden, P. Aoki, E. Cutrell, R. Jeffries, and G. Olson, Eds. New York, NY, USA: ACM, 04222006, pp. 979-988.

[69] W. Cui, S. Liu, L. Tan, C. Shi, Y. Song, Z. J. Gao, X. Tong, and H. Qu, "TextFlow: Towards Better Understanding of Evolving Topics in Text," IEEE Transactions on Visualization and Computer Graphics, vol. 17, no. 12, pp. 2412-2421, 2011.

[70] D. Gotz and H. Stavropoulos, "DecisionFlow: Visual Analytics for HighDimensional Temporal Event Sequence Data," IEEE Transactions on Visualization and Computer Graphics, vol. 20, no. 12, pp. 1783-1792, 2014.

[71] Y. Han, A. Rozga, N. Dimitrova, G. D. Abowd, and J. Stasko, "Visual Analysis of Proximal Temporal Relationships of Social and Communicative Behaviors," Computer Graphics Forum, vol. 34, no. 3, pp. 51-60, 2015.

[72] S. Liu, J. Yin, X. Wang, W. Cui, K. Cao, and J. Pei, "Online Visual Analytics of Text Streams," IEEE Transactions on Visualization and Computer Graphics, vol. 22, no. 11, pp. 2451-2466, 2016.

[73] M. El-Assady, R. Sevastjanova, D. A. Keim, and C. Collins, "ThreadReconstructor: Modeling Reply-Chains to Untangle Conversational Text through Visual Analytics," Computer Graphics Forum, vol. 37, no. 3, pp. 351-365, 2018.
[74] S. Fu, J. Zhao, H. F. Cheng, H. Zhu, and J. Marlow, "T-Cal: Understanding Team Conversation Data with Calendar-based Visualization," in Proceedings of the 36th CHI Conference on Human Factors in Computing Systems, ser. CHI, R. Mandryk, M. Hancock, M. Perry, and A. Cox, Eds. New York, NY, USA: ACM, 2018, pp. 1-13.

[75] C. Stoiber, A. Rind, F. Grassinger, R. Gutounig, E. Goldgruber, M. Sedlmair, S. Emrich, and W. Aigner, "Netflower: Dynamic Network Visualization for Data Journalists," Computer Graphics Forum, vol. 38, no. 3, pp. 699-711, 2019.

[76] Q. Li, Z. Wu, L. Yi, K. Seann, H. Qu, and X. Ma, "WeSeer: Visual Analysis for Better Information Cascade Prediction of WeChat Articles," IEEE Transactions on Visualization and Computer Graphics, vol. 26, no. 2, pp. 1399-1412, 2020.

[77] S. Hadlak, H. Schumann, C. H. Cap, and T. Wollenberg, "Supporting the Visual Analysis of Dynamic Networks by Clustering Associated Temporal Attributes," IEEE Transactions on Visualization and Computer Graphics, vol. 19, no. 12, pp. 2267-2276, 2013.

[78] M. Brehmer, S. Ingram, J. Stray, and T. Munzner, "Overview: The Design, Adoption, and Analysis of a Visual Document Mining Tool for Investigative Journalists," IEEE Transactions on Visualization and Computer Graphics, vol. 20, no. 12, pp. 2271-2280, 2014.

[79] E. Hoque and G. Carenini, "ConVis: A Visual Text Analytic System for Exploring Blog Conversations," Computer Graphics Forum, vol. 33, no. 3, pp. 221-230, 2014.

[80] N. Cao, C. Shi, S. Lin, J. Lu, Y.-R. Lin, and C.-Y. Lin, "TargetVue: Visual Analysis of Anomalous User Behaviors in Online Communication Systems," IEEE Transactions on Visualization and Computer Graphics, vol. 22, no. 1, pp. 280-289, 2016.

[81] Y. Lu, M. Steptoe, S. Burke, H. Wang, J.-Y. Tsai, H. Davulcu, D. Montgomery, S. R. Corman, and R. Maciejewski, "Exploring Evolving Media Discourse Through Event Cueing," IEEE Transactions on Visualization and Computer Graphics, vol. 22, no. 1, pp. 220-229, 2016.

[82] B. C. Kwon, S.-H. Kim, S. Lee, J. Choo, J. Huh, and J. S. Yi, "VisOHC: Designing Visual Analytics for Online Health Communities," IEEE Transactions on Visualization and Computer Graphics, vol. 22, no. 1, pp. 71-80, 2016.

[83] S. Fu, J. Zhao, W. Cui, and H. Qu, "Visual Analysis of MOOC Forums with iForum," IEEE Transactions on Visualization and Computer Graphics, vol. 23, no. 1, pp. 201-210, 2017.

[84] M. Khayat, M. Karimzadeh, J. Zhao, and D. S. Ebert, "VASSL: A Visual Analytics Toolkit for Social Spambot Labeling," IEEE Transactions on Visualization and Computer Graphics, vol. 26, no. 1, pp. 874-883, 2020.

[85] J. Zhao, F. Chevalier, C. Collins, and R. Balakrishnan, "Facilitating Discourse Analysis with Interactive Visualization," IEEE Transactions on Visualization and Computer Graphics, vol. 18, no. 12, pp. 2639-2648, 2012.

[86] N. Cao, Y.-R. Lin, X. Sun, D. Lazer, S. Liu, and H. Qu, "Whisper: Tracing the Spatiotemporal Process of Information Diffusion in Real Time," IEEE Transactions on Visualization and Computer Graphics, vol. 18, no. 12, pp. 2649-2658, 2012.

[87] M. El-Assady, V. Gold, C. Acevedo, C. Collins, and D. A. Keim, "ConToVi: Multi-Party Conversation Exploration using Topic-Space Views," Computer Graphics Forum, vol. 35, pp. 431-440, 2016. [Online]. Available: http://doi.wiley.com/10.1111/cgf.12919

[88] S. K. Card, J. D. Mackinlay, and B. Shneiderman, Readings in Information Visualization: Using Vision to Think, ser. Morgan Kaufmann Series in Interactive Technologies. San Francisco, CA: Morgan Kaufmann, 1999.

[89] H.-W. Ng, V. D. Nguyen, V. Vonikakis, and S. Winkler, "Deep Learning for Emotion Recognition on Small Datasets using Transfer Learning," in Proceedings of the 2015 ACM on International Conference on Multimodal Interaction, Z. Zhang, P. Cohen, D. Bohus, R. Horaud, and H. Meng, Eds. New York: ACM, 2015, pp. 443-449.

[90] A. Sarikaya, M. Gleicher, and D. A. Szafir, "Design Factors for Summary Visualization in Visual Analytics," Computer Graphics Forum, vol. 37, no. 3, pp. 145-156, 2018.

[91] J. S. Yi, Y. A. Kang, J. Stasko, and J. Jacko, "Toward a Deeper Understanding of the Role of Interaction in Information Visualization," Transactions on Visualization and Computer Graphics, vol. 13, no. 6, pp. 1224-1231, 2007.

[92] T. Spinner, U. Schlegel, H. Schafer, and M. El-Assady, "explAIner: A Visual Analytics Framework for Interactive and Explainable Machine Learning," IEEE Transactions on Visualization and Computer Graphics, vol. 26, no. 1, pp. 1064-1074, 2020. 


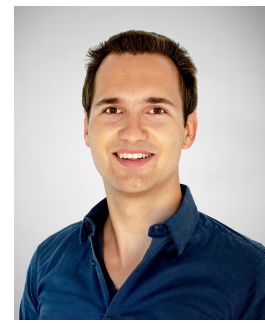

Maximilian T. Fischer is a research associate and $\mathrm{PhD}$ candidate at the Data Analysis and Visualization Research Group at the University of Konstanz, Germany. He received his M.Sc. degree in Computer and Information Science from the University of Konstanz in 2019. His research interests include the interactive visualization and analysis of communication as well as multivariate and temporal hypergraph modeling.

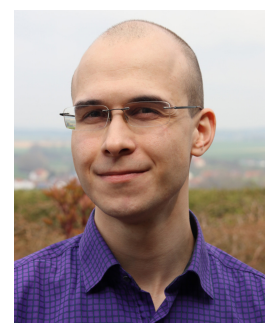

Frederik L. Dennig is a PhD candidate at the Data Analysis and Visualization Research Group at the University of Konstanz, Germany, since 2019. His research interests include the featurebased analysis of subspaces as well as pattern detection and similarity search.

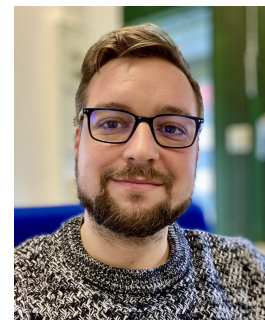

Daniel Seebacher is a PhD candidate at the Data Analysis and Visualization Research Group at the University of Konstanz, Germany, since 2016. His research interests include the visual analysis of spatio-temporal events and their context. Concrete research domains include the analysis of the spread of invasive species, the study of intra-city meteorological phenomena, or the study of actions and interactions in sports such as soccer or tennis.

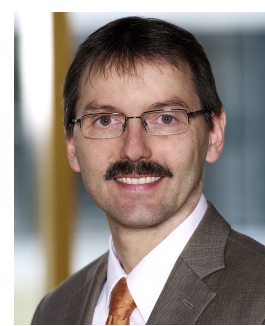

Daniel A. Keim is professor and head of the Data Analysis and Visualization Research Group in the Computer Science Department at the University of Konstanz, Germany. He has been actively involved in data analysis and information visualization research for more than 25 years and developed novel visual analysis techniques for large data sets. Dr. Keim got his Ph.D. degree in Computer Science from the University of Munich, Germany. Before joining the University of Konstanz, Dr. Keim was associate professor at the University of Halle, Germany, and Technology Consultant at AT\&T Shannon Research Labs, NJ, USA.

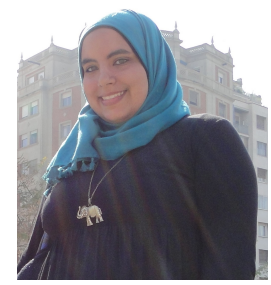

Mennatallah El-Assady is a research associate in the Data Analysis and Visualization Research Group at the University of Konstanz, Germany, and in the Visualization for Information Analysis lab at the University of Ontario Institute of Technology, Canada. She received her M.Sc. degree in Information Engineering from the University of Konstanz in 2015. Her research interests include visual text analytics, user-steerable topic modeling, and discourse/conversational text analysis. 